\title{
Sensitivity analysis of a relative navigation solution for unmanned aerial vehicles in a GNSS-denied environment
}

Jeremy Hardy

Follow this and additional works at: https://researchrepository.wvu.edu/etd

\section{Recommended Citation}

Hardy, Jeremy, "Sensitivity analysis of a relative navigation solution for unmanned aerial vehicles in a GNSS-denied environment" (2016). Graduate Theses, Dissertations, and Problem Reports. 5763.

https://researchrepository.wvu.edu/etd/5763

This Thesis is protected by copyright and/or related rights. It has been brought to you by the The Research Repository @ WVU with permission from the rights-holder(s). You are free to use this Thesis in any way that is permitted by the copyright and related rights legislation that applies to your use. For other uses you must obtain permission from the rights-holder(s) directly, unless additional rights are indicated by a Creative Commons license in the record and/ or on the work itself. This Thesis has been accepted for inclusion in WVU Graduate Theses, Dissertations, and Problem Reports collection by an authorized administrator of The Research Repository @ WVU. For more information, please contact researchrepository@mail.wvu.edu. 


\title{
SENSITIVITY ANALYSIS OF A RELATIVE NAVIGATION SOLUTION FOR UNMANNED AERIAL VEHICLES IN A GNSS-DENIED ENVIRONMENT
}

\author{
Jeremy Hardy
}

\author{
Thesis submitted to the \\ Benjamin M. Statler College of Engineering and Mineral Resources \\ at West Virginia University \\ in partial fulfillment of the requirements for the degree of \\ Master of Science \\ in \\ Aerospace Engineering
}
Jason N. Gross, Chair Ph.D.
John A. Christian, Ph.D.
Yu Gu, Ph.D.

Department of Mechanical and Aerospace Engineering

Morgantown, West Virginia
2016

Keywords: GPS-Denied Navigation, Relative Inertial Navigation, Sensor Fusion

Copyright (C) 2016 


\title{
ABSTRACT \\ Sensitivity Analysis of a Relative Navigation Solution for Unmanned Aerial Vehicles in a GNSS-denied Environment
}

\author{
Jeremy Hardy
}

Cooperative navigation between two or more unmanned aerial vehicles (UAVs) is an important enabling technology for problems such as military reconnaissance, disaster response, and search and rescue. In many of these situations Global Navigation Satellite Systems (GNSS), such as Global Positioning System (GPS), may be unreliable or unavailable due to structural impedance or malicious signal jamming. Therefore, the task of maintaining a reliable relative navigation solution without the use of GNSS is an important need for the aforementioned missions.

To meet this need, this thesis focuses on the relative navigation between two UAVs that are operating in a GNSS-denied environment. In particular, the design and sensitivity of a navigation algorithm are presented. The navigation algorithm presented consists of an Unscented Kalman filter that fuses multiple on-board sensors to estimate the relative pose between two UAVs. These sensors include: strap-down inertial measurement units, ultra-wideband ranging radios, strap-down triaxial magnetometers, and downward facing cameras. Through the use of a Monte Carlo simulation study, the presented algorithm's performance sensitivity to various sensor payload characteristics, flight dynamics, and initial condition errors is evaluated. Additionally, a research platform that will provide for a future experimental evaluation of the algorithm presented in this thesis has been integrated and tested as part of this work. 


\section{Acknowledgments}

First, I would like to thank my research advisor and thesis committee chair Dr. Jason Gross. His guidance, knowledge, and continued support made this thesis possible and has molded me into the student I am today. He made this opportunity possible for me and I am sincerely grateful. I would also like to thank my thesis committee members Dr. Yu Gu and Dr. John Christian. Their valued advice and knowledge was crucial to the work presented in this thesis.

I would also like to acknowledge the Air Force Research Laboratory (AFRL) and the National Geospacial-Intelligence Agency (NGA). The funding provided by these two agencies allowed me to work on projects relevant to my interests while also providing an affordable way to continue my education. I would like to thank Systems and Technology Research (STR) for the work provided as a partner on the AFRL research grant. Their work was instrumental to the completion of the thesis. Additionally, I would like to thank them for the opportunity to work as a summer intern, and greatly look forward to my return as a full time employee.

Finally, I would like to thank my family and my girlfriend for their loving support through these years of study, I couldn't have done this without you! 


\section{Contents}

$\begin{array}{ll}\text { Abstract } & \text { ii }\end{array}$

Acknowledgments $\quad$ iv

List of Figures $\quad$ vii

List of Tables $\quad$ ix

1 Introduction 1

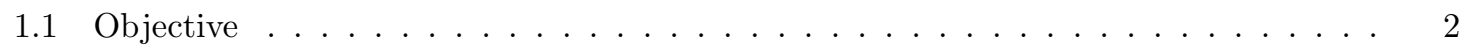

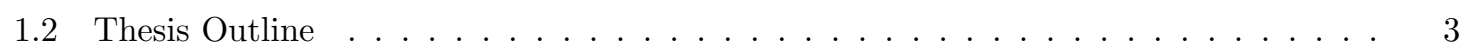

2 Problem Statement $\quad 4$

3 Relative Inertial Navigation $\quad 9$

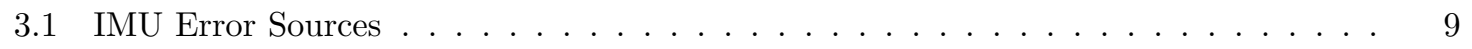

$3.1 .1 \quad$ Scale Factor . . . . . . . . . . . . . . . . . . . . . 9

3.1 .2 Non-orthogonality . . . . . . . . . . . . . . . . . . . 9

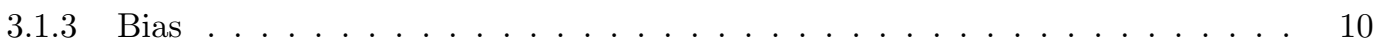

3.1.4 Random Walk . . . . . . . . . . . . . . . . . . . . . . . . 10

3.2 Inertial Measurement Unit . . . . . . . . . . . . . . . . . . . . . . . . 10

3.3 Relative Quaternion Update . . . . . . . . . . . . . . . . . . . . . . . . . . . . . . . 10

3.3.1 Required Attitude Transformation Mathematics . . . . . . . . . . . . . 11

3.4 Relative Velocity Update . . . . . . . . . . . . . . . . . . . . . . . . . 13

3.5 Relative Position Update . . . . . . . . . . . . . . . . . . . . . . 14

4 Computer Vision Algorithm $\quad 15$ 


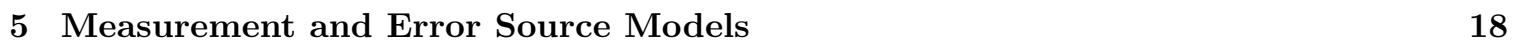

5.1 Ultra-Wideband Ranging Radio . . . . . . . . . . . . . . . . . . . . . . . 18

5.2 Tri-axial Magnetometers . . . . . . . . . . . . . . . . . . . . . . . . 18

5.3 Computer Vision . . . . . . . . . . . . . . . . . . . . . . . . . . . 19

5.3.1 Rotation Element ....................... 20

5.3.2 Unit Vector Element ........................ 21

6 Unscented Kalman Filter $\quad 22$

6.1 Unscented Transform . . . . . . . . . . . . . . . . . . . . . . . . . . . . 22

6.2 Prediction Transformation . . . . . . . . . . . . . . . . . . . . . 23

6.3 Observation Transformation . . . . . . . . . . . . . . . . . . . . . . . . 24

6.4 Measurement Update . . . . . . . . . . . . . . . . . . . . . . . 24

6.5 Assigning Process and Measurement Noise . . . . . . . . . . . . . . . . . . 25

7 Simulation and Monte Carlo 29

7.1 Monte Carlo Design . . . . . . . . . . . . . . . . . . . . . . . . 29

7.2 Monte Carlo Results . . . . . . . . . . . . . . . . . . . . . . . . . . . 31

7.3 Single Simulation Results . . . . . . . . . . . . . . . . . . . . . . . . . 43

8 Experimental Platform $\quad 48$

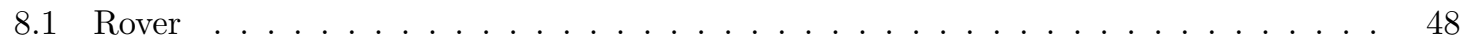

8.2 Quadrotor .............................. 49

8.3 UWB Protocol . . . . . . . . . . . . . . . . . . . . . . . . 50

8.4 System Overview . . . . . . . . . . . . . . . . . . . . . . . 51

9 Conclusion $\quad 54$ 


\section{List of Figures}

2.1 Diagram of Sensor Payloads and Assumptions . . . . . . . . . . . . . . . . . 4

2.2 Three Dimensional Representation of Flight Path $1 \ldots \ldots \ldots$

2.3 Three Dimensional Representation of Flight Path $2 \ldots \ldots \ldots$

2.4 Three Dimensional Representation of Flight Path $3 \ldots \ldots \ldots$

2.5 Magnetic Field Assumption Check for Flight Path $1 \ldots \ldots$. . . . . . . . 7

2.6 Magnetic Field Assumption Check for Flight Path $2 \ldots \ldots \ldots$

2.7 Magnetic Field Assumption Check for Flight Path $3 \ldots \ldots$. . . . . . . . 8

4.1 Computer Vision Algorithm Visual Representation . . . . . . . . . . . . . . . . 15

4.2 Latent Dirichlet Plate Notation Model . . . . . . . . . . . . . . . . . . . . . 17

5.1 Distribution of Computer Vision Rotation Error _ . . . . . . . . . . . . . 20

5.2 Distribution of Computer Vision Unit Vector Error ～. . . . . . . . . . . . . . . 21

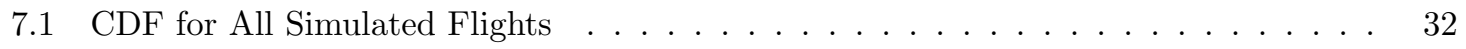

7.2 CDF for Inertial Measurement Unit Quality . . . . . . . . . . . . . . . . . 33

7.3 CDF for Inertial Measurement Unit Quality . . . . . . . . . . . . . . . . . . 33

7.4 CDF for Flight Path Selections . . . . . . . . . . . . . . . . . . . . . 34

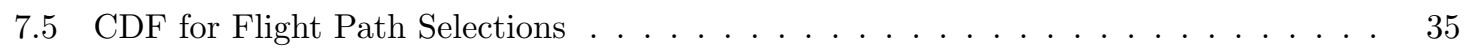

7.6 CDF for UWB Ranging Radio Measurement Frequency _ . . . . . . . . . . . . 36

7.7 CDF for UWB Ranging Radio Measurement Frequency $\ldots \ldots \ldots$

7.8 CDF for UWB Ranging Radio Measurement Error Scale Factor . . . . . . . . . . . . 37

7.9 CDF for UWB Ranging Radio Measurement Error Scale Factor . . . . . . . . . . . . 38

7.10 CDF for Magnetometer Measurement Frequency . . . . . . . . . . . . . . 39

7.11 CDF for Magnetometer Measurement Frequency . . . . . . . . . . . . . . 39

7.12 CDF for Magnetometer Measurement Error Scale Factor . . . . . . . . . . . . . . 40 
7.13 CDF for Magnetometer Measurement Error Scale Factor . . . . . . . . . . . . . . . . 41

7.14 CDF for Computer Vision Measurement Frequency . . . . . . . . . . . . . . . . . . . 42

7.15 CDF for Computer Vision Measurement Frequency . . . . . . . . . . . . . . . . . . 42

7.16 Comparison of Navigation Algorithm and IMU Only for Position Estimate . . . . . . 44

7.17 Comparison of Navigation Algorithm and IMU Only for Velocity Estimate . . . . . . 44

7.18 Comparison of Navigation Algorithm and IMU Only for Attitude Estimate . . . . . 45

7.19 Comparison of Navigation Algorithm and IMU Only for Attitude Estimate . . . . . 46

7.20 UKF Solution Compared to Truth in Beginning of Flight . . . . . . . . . . . . . . . 47

7.21 UKF Solution Compared to Truth after Aircraft Convergence . . . . . . . . . . . . . 47

8.1 Rover with Visible UWB Radio and GPS Antennas at the Top of Mast . . . . . . . 49

8.2 Quadrotor with Visible UWB Radio and GPS Antennas . . . . . . . . . . . . . . . 50

8.3 UWB Ranging Radio and NovAtel Pinwheel Antenna on Rover Mast . . . . . . . . . 51

8.4 Flight Testing Photo before Quadrotor Takeoff . . . . . . . . . . . . . . . . . . 52

8.5 Flight Testing Photo with Quadrotor in Flight . . . . . . . . . . . . . . . 53 


\section{List of Tables}

3.1 IMU Grade Scale Factors $\ldots \ldots \ldots \ldots$

5.1 Statistics of Distribution for Rotational Component of Computer Vision Measurements 20

5.2 Statistics of Distribution for Unit Vector Component of Computer Vision Measurements 21

7.1 Distribution of IMU Quality into Monte Carlo Analysis . . . . . . . . . . . . . 29

7.2 Distribution of Flight Paths into Monte Carlo Analysis . . . . . . . . . . . . . . 30

7.3 Distribution of Ranging Radio Frequencies into Monte Carlo Analysis . . . . . . . 30

7.4 Distribution of Ranging Radio Error Scale Factors into Monte Carlo Analysis . . . 30

7.5 Distribution of Magnetometer Frequencies into Monte Carlo Analysis . . . . . . . . . 30

7.6 Distribution of Magnetometer Error Scale Factors into Monte Carlo Analysis . . . 31

7.7 Distribution of Computer Vision Frequencies into Monte Carlo Analysis . . . . . . . 31

7.8 Distribution of Initial Error for Each State $\ldots \ldots \ldots \ldots$

7.9 Median RSS Error Values of All Monte Carlo Flights . . . . . . . . . . . . . . . . 32

7.10 Mean RSS Error Values at Several Percentages of Monte Carlo Flights . . . . . . . . 43 


\section{Acronyms}

$\begin{array}{ll}\text { AFRL } & \text { Air Force Research Laboratory } \\ \text { ARW } & \text { Angular Random Walk } \\ \text { CDF } & \text { Cumulative Distribution Function } \\ \text { EKF } & \text { Extended Kalman Filter } \\ \text { GNSS } & \text { Global Navigation Satellite System } \\ \text { GPS } & \text { Global Positioning System } \\ \text { HMM } & \text { Hidden Markov Model } \\ \text { IMU } & \text { Inertial Measurement Unit } \\ \text { INS } & \text { Inertial Navigation System } \\ \text { LDA } & \text { Latent Dirichlet Allocation } \\ \text { MAV } & \text { Minature Air Vehicles } \\ \text { NGA } & \text { National Geospacial-Intelligence Agency } \\ \text { RANSAC } & \text { Random Sampling and Conseneus } \\ \text { RSS } & \text { Root Sum of Squares } \\ \text { SIFT } & \text { Scale Invariance Feature Transform } \\ \text { SLAM } & \text { Simultaneous Localization and Mapping } \\ \text { STR } & \text { System and Technology Research } \\ \text { UAV } & \text { Unmanned Aerial Vehicle } \\ \text { UKF } & \text { Unscented Kalman Filter } \\ \text { UWB } & \text { Ultra-Wideband } \\ \text { VRW } & \text { Velocity Random Walk } \\ & \end{array}$




\section{Chapter 1}

\section{Introduction}

In recent years cooperative navigation techniques have been applied to a multitude of problems as the benifits of using two or more aircraft to cover large areas in applications such as search and rescue, cave or tunnel mapping, disaster response, and military survailence missions is invaluable. Methods for accurately and efficiently mapping or searching an area through cooperative methods has been extensively researched and is demonstrated in a multitude of works[1], [2],[3], [4], [5].

Several areas in which these cooperative methods are most useful are also areas of application in which GNSS may be unavailable or degraded, either through malicious jamming, or unfortunate enviornmental conditions, such as caves, collapsed buildings, or urban canyon environments. As most modern methods for navigation use GNSS updates to reduce the known drift in Inertial Navigation System (INS) dead reackoning solutions [6], alternatives to GNSS for these applications must be developed.

A popular solution to navigating in a GNSS-denied environment is to use image based navigation to update INS. Visual odemetry using feature tracking and image registration along side INS and barametric pressure sensors was able to improve INS only solution when GNSS was assumed to be unavailable in [7].

Additional visual methods such as Simultanious Localization and Mapping (SLAM), which can also be applied to infrared and laser based systems, has also shown to be a viable method for correcting or stabalizing INS drift when GNSS is not available and is particularly advantegous when the vehicle is known to return to its starting position, closing a "loop" [8],[9],[10],[11].

A relative approach to estimating pose through SLAM is implimented in [12] where position and yaw states are estimated with respect to the current keyframe, or image reference, of the visual 
SLAM algorithm.

Vision techniques are also applied to relative navigation of UAVs for formation flight and air refueling are descibed in [13] and [14]. These applications have a single camera on-board an aircraft navigating to a fueling dock or following another aircraft. Both of these aplications require line of sight to the object of relative interest and [13] uses cooperative flight planning in one of the solutions presented.

A navigation solution for swarms of Minature Air Vehicles (MAVs) in a GNSS denied enviornment is explored in [15]. Where the solution requires two separate swarms of MAVs with the ability to start and stop in a cooperative manner. Instead of using popular vision techniques or line of sight sensing, the swarm of aircraft distribute INS data across all MAVs in the swarm in order to reduce dead reckoning drift as a group.

The kinematics of using relative inertial navigation are presented in [16], along with an algorithm designed for estimating relative state of two aircraft using three line of sight measurements from one aircraft to another. Cooperative navigation techniques are also applied to the formulation to optimize the observability of the line of sight measurements used in the estimation technique. One of the underlying assumptions of this paper is that the absolute state of one of the aircraft is known.

Similar to the aircraft to aircraft line of sight measurements in [16], Ultra-Wideband (UWB) peer-to-peer ranging radio measurements are implemented in [17] for close formation flight of small UAVs. The UWBs are included to make the differential GPS/INS solution robust to degregated GPS situations such as phase breaks or poor satellite geometry.

While several different sensors have already been discussed for use in relative navigation [18] presents an algorithm that is able to theoretically calculate relative pose of two UAVs using only INS and peer-to-peer ranging data. This algorithm was dimenstrated in $2 \mathrm{D}$ and the methods can be extrapolated to 3D. However, the algorithm requires very specific aircraft dynamics and coopererative manuevers to eliminate ambiguities. The work was presented as a way to initalize another algorithm in which the dynamics of each aircraft could be less constricted, specifically for [19] which is what this thesis most closely relates to, as much of the algorithm has remained unchanged since the writing of the conference paper.

\section{$1.1 \quad$ Objective}

The objective of this thesis is to present a relative navigation Unscencted Kalman Filter (UKF) within a simulated Global Navigation Satellite System (GNSS) denied environment by fusing INS, 
computer vision, UWB ranging radio, and magnetometer sensor data. Then characterize its sensitivity to various on-board sensor characteristics, flight dynamics, and initial condition errors. The algorithm and Monte Carlo set-up is reviewed before presenting and analyzing the results of the sensitivity analysis and a more in-depth look at an individual flight. Additionally, a description of the experimental platforms built along side the simulation work is presented as well as the potential for future testing it provides.

\subsection{Thesis Outline}

The second chapter of this thesis outlines the exact payload of the UAVs, the simulated flight paths used, and the underlying assumptions. The next chapter outlines the relative INS. Then, an overview of the computer vision algorithm is presented. Next, error and observation models for ranging radios, magnetometers, and computer vision measurements are detailed for this particular application. Chapter six displays the process of the UKF algorithm step by step. Chapter seven outlines the details of the simulation and Monte Carlo including the results of the study. Next, the experimental platform designed to test the algorithm is described. Finally, chapter nine provides some concluding remarks and plans for future work. 


\section{Chapter 2}

\section{Problem Statement}

Each aircraft payload includes a tri-axial Inertial Measurement Unit (IMU) and magnetometer, peer-to-peer UWB ranging radios, and a downward facing camera. The system also assumes perfect and simultaneous data transfer to whichever aircraft or ground system is estimating position and attitude, sometimes referred to as pose. In this thesis the follower aircraft is navigating to the leader, which is moving without restriction, and therefore would be the ideal platform for running the navigation algorithm.

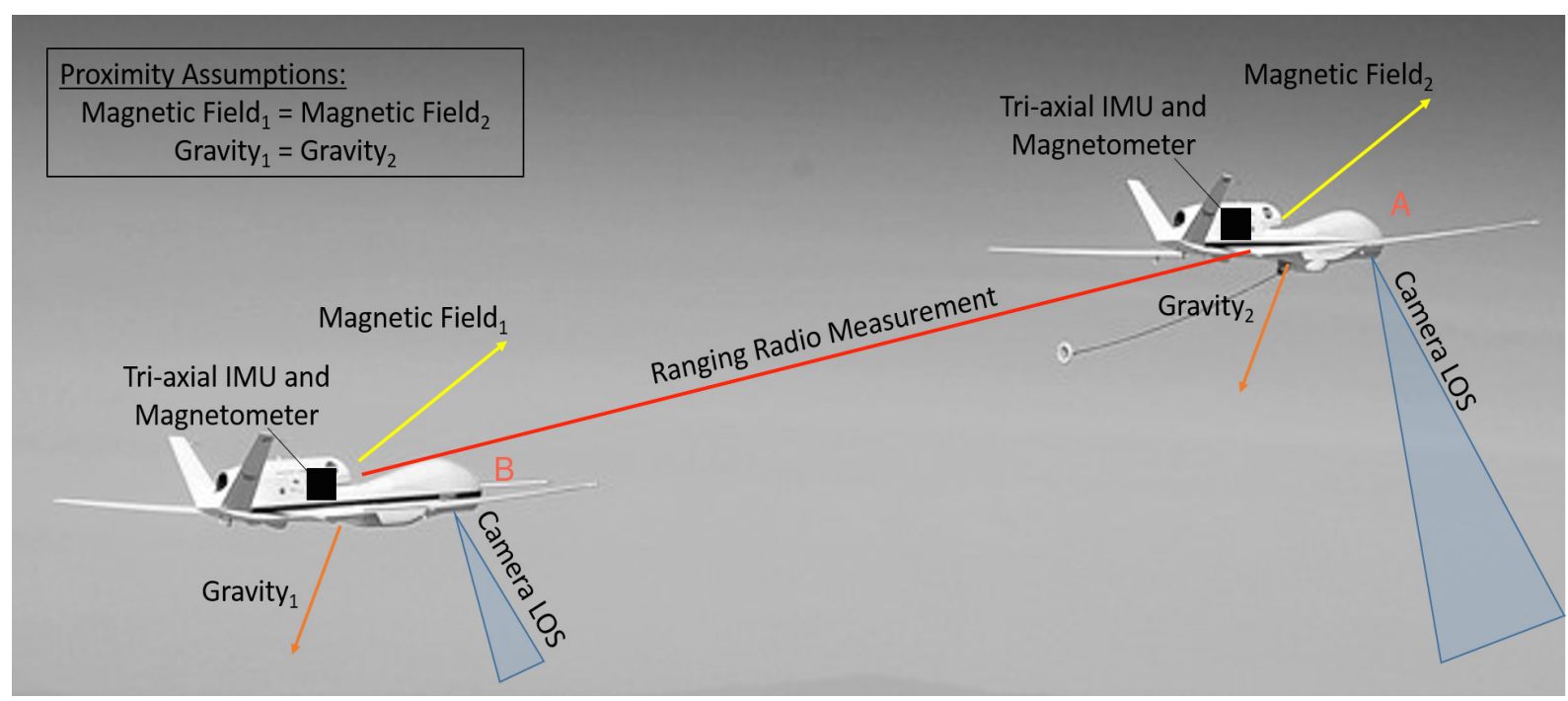

Figure 2.1: Diagram of Sensor Payloads and Assumptions

The estimated state vector is composed of: relative position, relative velocity, and relative attitude. All elements of the state vector are expressed as values from the leader aircraft, $l$, to the follower aircraft, $f$, resolved in the body frame of the follower. The resolving frame is expressed as 
a superscript, while the subscripts describe the relative nature of the variable, and is read as: from subscript 1 to subscript 2. Position, $r$, and attitude, $\theta$, represents the total relative pose between aircraft in meters and Euler angles.

$$
x=\left[\begin{array}{c}
r_{l f}^{f} \\
v_{l f}^{f} \\
\boldsymbol{\theta}_{l f}^{f}
\end{array}\right]
$$

The algorithms initial state estimates are provided by either a recent, reliable, GNSS update of both aircraft, or through cooperative methods for pose calculation like those found in [18]. The flight paths used for testing begin at altitude with varying distances between the aircraft. The leader aircraft is operating with no restriction in an oval path for approximately eight minutes. The follower aircraft begins at three different positions with relative position magnitudes ranging from approximately 15 meters to over 2000 meters. The follower aircraft then maneuvers toward the leader over a period of time.

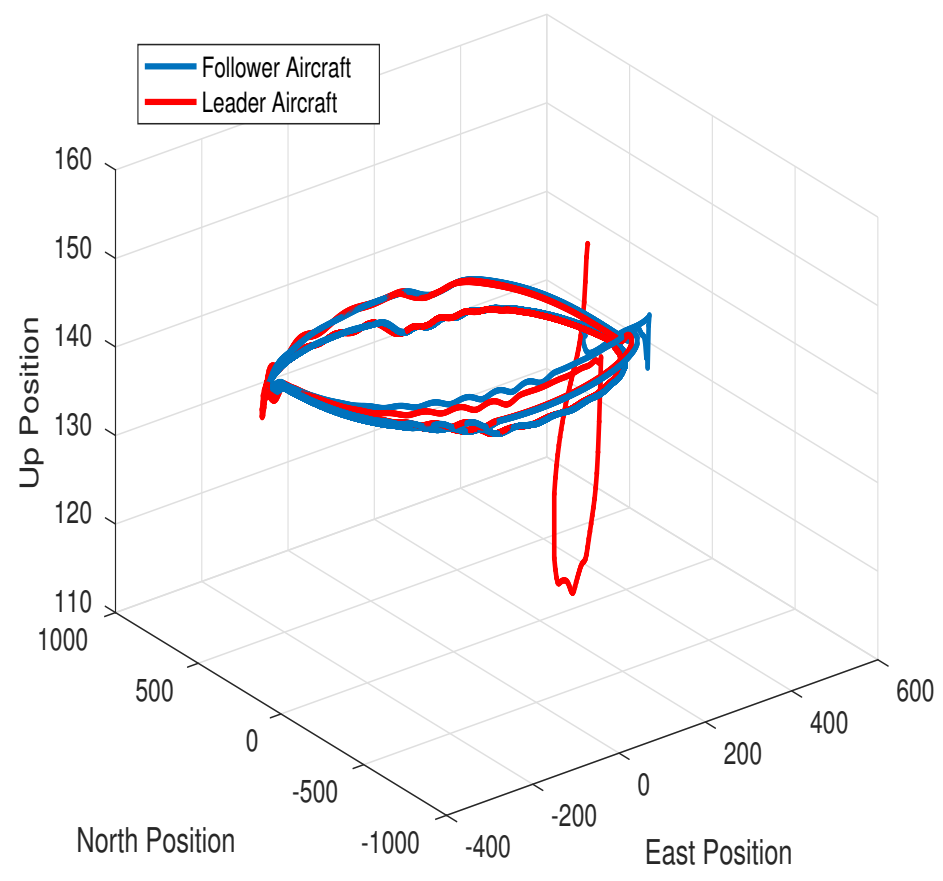

Figure 2.2: Three Dimensional Representation of Flight Path 1 


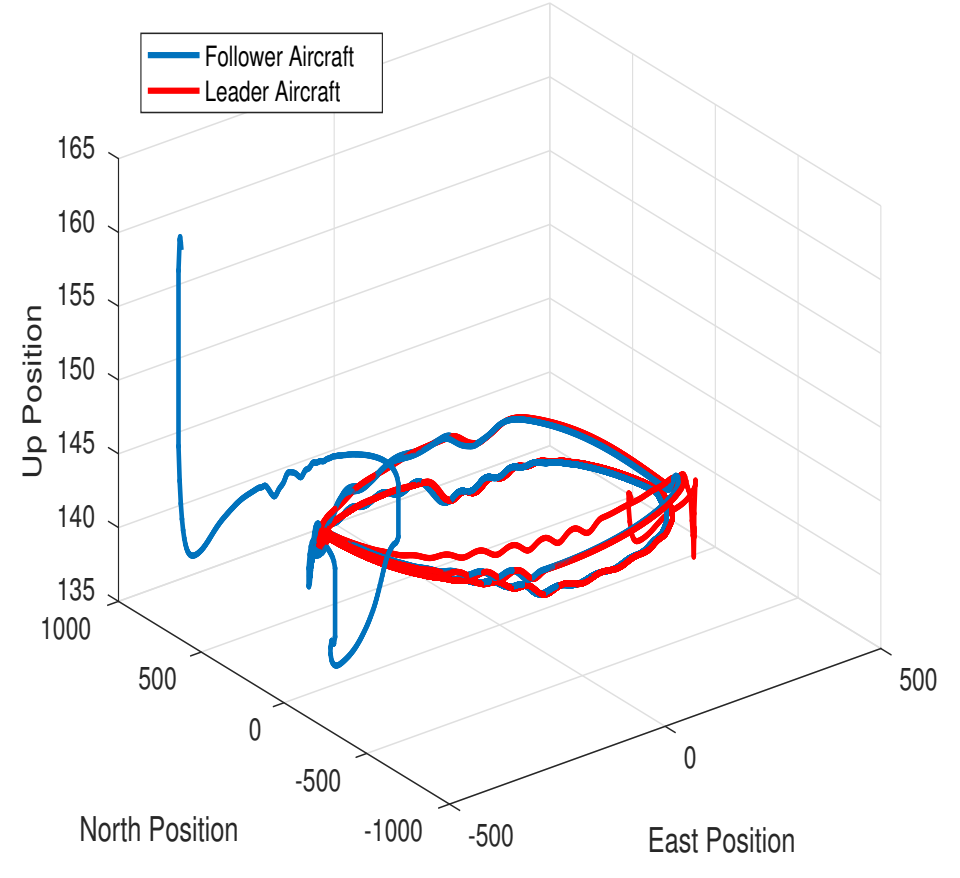

Figure 2.3: Three Dimensional Representation of Flight Path 2

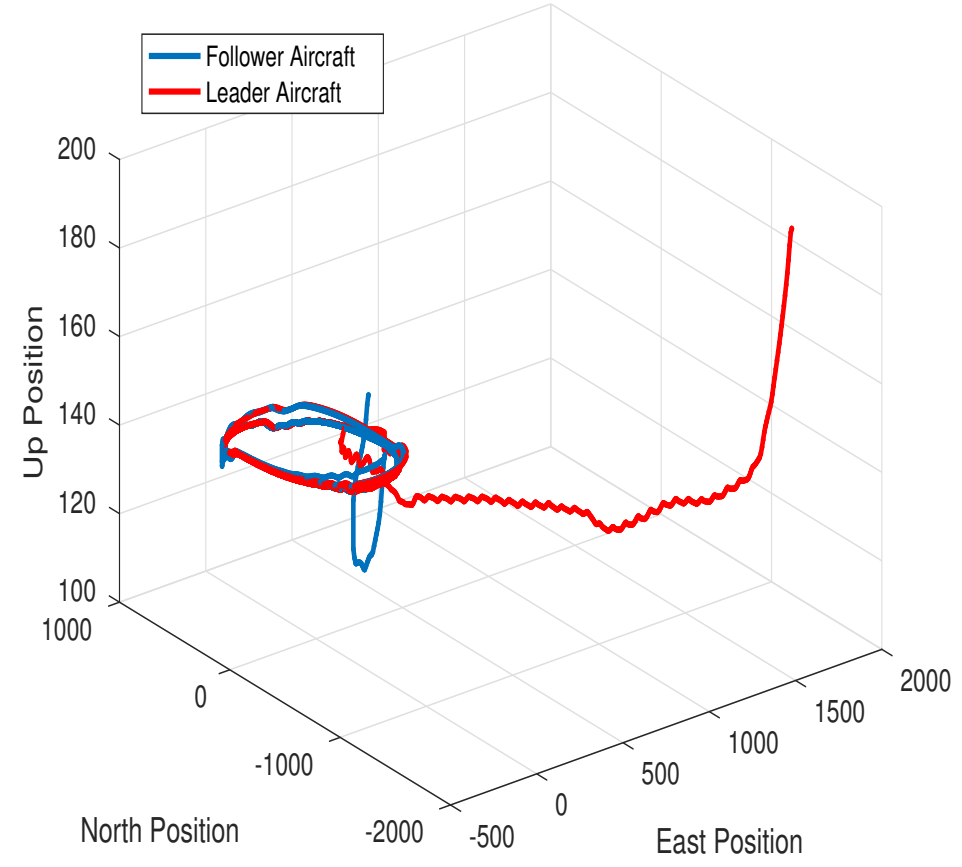

Figure 2.4: Three Dimensional Representation of Flight Path 3 
Larger starting distance between aircraft results in a longer period of dynamic flight before the follower aircraft can reach the leader. Comparing Figures 2.2 and 2.4, where the starting positions are the most extreme, the difference in flight dynamics and the time it takes the follower aircraft to converge to leaders position is substantial.

The navigation solution described in this thesis is specifically constructed for two identical aircraft operating in proximity to one another. This is important to note as assumptions play a large role in the functionality of the presented algorithm. The proximity of the aircraft allows for a reasonable assumption of shared natural magnetic field and gravitational forces as both phenomena generally experience negligible changes across short distances.

Identical aircraft operating in proximity allows for the assumption to be made that the only substantial differences in tri-axial magnetometer readings on each aircraft will be a result of differences in aircraft attitude. As a way of displaying this assumption, the true magnetic field forces from the simulation where transformed with the true rotation matrix to compare to leader aircraft magnetometer to follower aircraft magnetometer without error sources. Figures $2.5-2.7$ shows the results of Equation 2.2 for each of the three flight paths, where $M_{f, l}$ are true magnetic field values and $R_{l}^{f}$ is the true rotation from the body frame of the leader aircraft to the body frame of the follower aircraft.

$$
M_{\text {difference }}=\frac{M_{f}-R_{l}^{f}\left(M_{l}\right)}{\left\|M_{f}\right\|_{2}} \times 100
$$

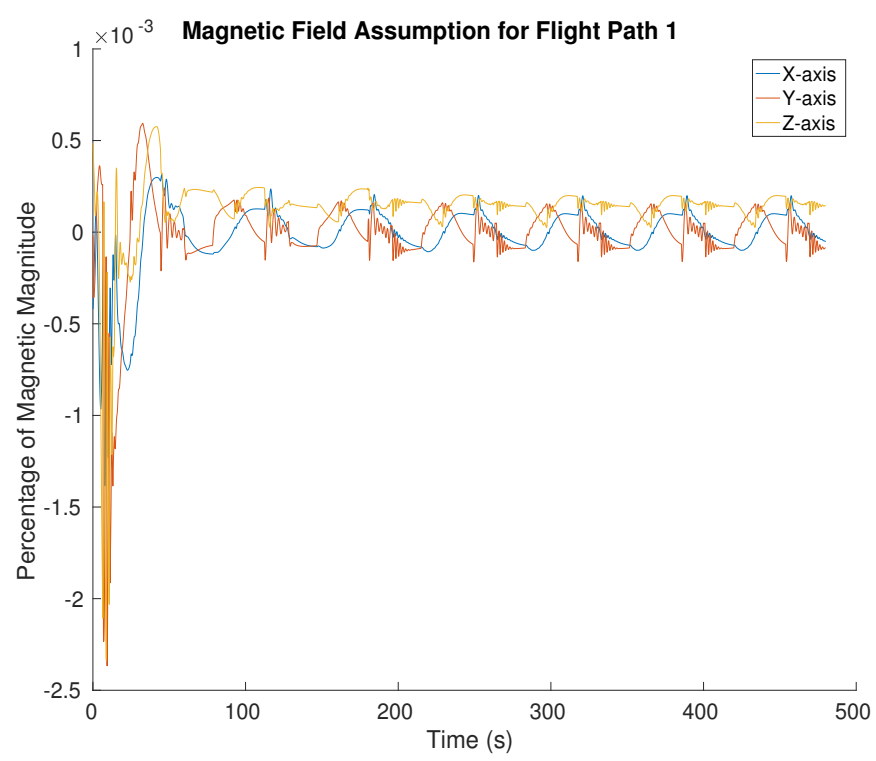

Figure 2.5: Magnetic Field Assumption Check for Flight Path 1 


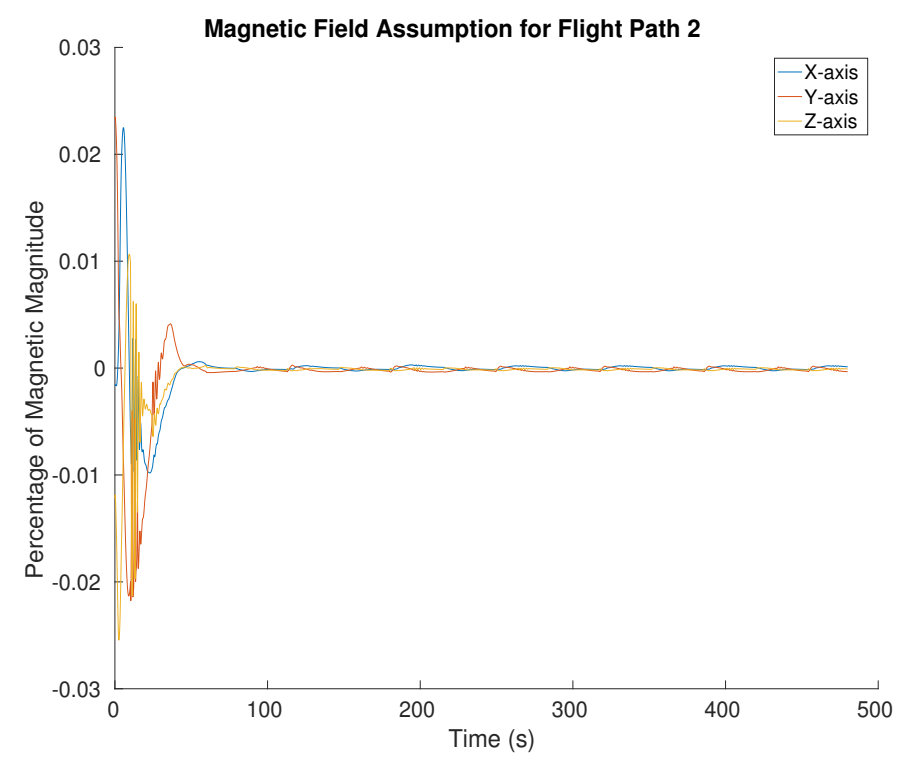

Figure 2.6: Magnetic Field Assumption Check for Flight Path 2

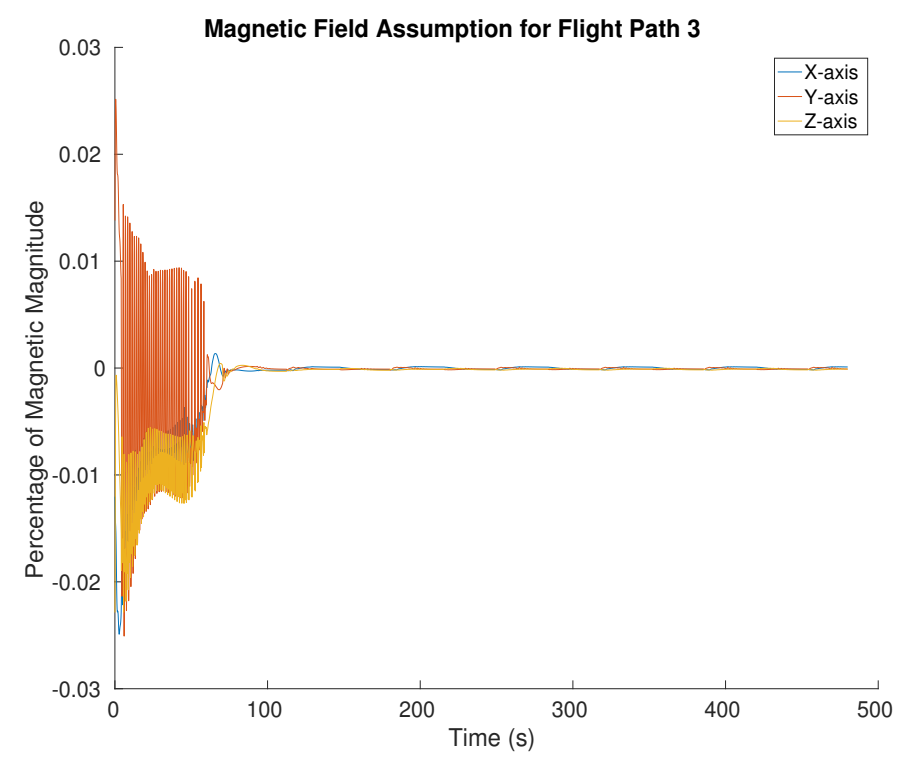

Figure 2.7: Magnetic Field Assumption Check for Flight Path 3

Comparing Figures 2.5 and 2.7, the flights with the smallest and largest starting aircraft distances, respectively, the effect that distance has on the magnetic field assumption is apparent. However, even at the largest distance between aircraft the maximum difference between magnetic field values of the two aircraft as a percentage of the magnetic field magnitude is less than $0.03 \%$, confirming that the magnetic field assumption is viable at these tested distances. 


\section{Chapter 3}

\section{Relative Inertial Navigation}

This chapter reviews the error sources present in IMU data [6], the characteristics of the IMUs used in this thesis, and the process used to propagate the relative state forward one discrete time step.

\subsection{IMU Error Sources}

Strap-down IMUs are composed of three accelerometers and three gyroscopes aligned orthogonally and fixed to the body in which the user is interested in. Accelerometers report data as specific force or incremental change in velocity, $\Delta v$. While gyroscopes report data as angular rate or incremental change in angular position, $\Delta \theta[6],[20]$.

\subsubsection{Scale Factor}

Scale factor error is error in the value used to map sensor values to the actual values they are meant to represent. Often times this means multiplying an output voltage by a scalar to produce the correct scale and units of the desired output from the sensor. Any error in the factor required to acquire the proper value obviously relates to error in the final reading be used.

\subsubsection{Non-orthogonality}

Due to limitations in manufacturing, the sensors are never perfectly aligned at $90^{\circ}$ as they are intended to be. This results in correlation in measurements from one axis to another. This error can often be negated through careful calibration. 


\subsubsection{Bias}

Bias appears in two forms in IMU measurements. First, a turn on bias is a constant difference between the truth value and readings of the accelerometer or gyroscope in an axis of the IMU. Second, IMUs experience measurement drift, described as in-run stability biases of either accelerometer or gyroscopes, is the drift in the turn on bias over time.

\subsubsection{Random Walk}

Random walk is an error source that arises from the integration of sensor white noise. Noisy readings from both gyroscopes and accelerometers are integrated to calculate attitude and velocity and thus result in Angular Random Walk (ARW) and Velocity Random Walk (VRW), respectively.

\subsection{Inertial Measurement Unit}

In this thesis IMU measurments are modeled using only the effects of white noise, in-run stability and random walk error sources. The Honeywell HG1930-CA50 was used as the baseline IMU in this thesis. The sensor has a gyroscope bias in-run stability of $1.0 \frac{\mathrm{deg}}{\mathrm{hr}}$, a accelerometer bias in-run stability of $0.3 \mathrm{mg}$, a Velocity Random Walk (VRW) of $0.3 \frac{\mathrm{fps}}{\sqrt{h r}}$ and an Angular Random Walk (ARW) of $0.125 \frac{\mathrm{deg}}{\sqrt{h r}}$ in the roll axis and $0.09 \frac{\mathrm{deg}}{\sqrt{h r}}$ in the pitch and yaw axes [21]. Scaling factors where then applied to these characteristics to match various grade IMU characteristics ranging from Automotive to Aviation applications as presented in [6]. As the baseline IMU fell into the range of Tactical IMU the scale factors in Table 3.1 are used to generate the other grades of IMUs.

\begin{tabular}{|l|c|}
\hline IMU Characterization & Error Scale Factor \\
\hline Automotive Grade & 50 \\
\hline Tactical Grade & 1 \\
\hline Intermediate Grade & $1 / 100$ \\
\hline Aviation Grade & $1 / 1000$ \\
\hline
\end{tabular}

Table 3.1: IMU Grade Scale Factors

\subsection{Relative Quaternion Update}

Quaternion representation used in this thesis is from the work presented in [22], [16]. The representation of the relative quaternion can be found in Equation 3.1 and the vector formulation in 
Equation 3.2. It should be noted that in some literature $q_{4}$ can also be found as the first element of the vector.

$$
\begin{gathered}
q=i q_{1}+j q_{2}+k q_{3}+q_{4} \\
q=\left[\begin{array}{l}
q_{1} \\
q_{2} \\
q_{3} \\
q_{4}
\end{array}\right]
\end{gathered}
$$

Angular rate measurements are represented as $\omega_{f}$ and $\omega_{l}$, for the follower and leader platforms, respectively. The relative quaternion that maps the body frame of the leader platform to the body frame of the follower platform can then be propagated using Equation 3.3 [22] [16].

$$
q_{l f}^{f,+}=\bar{\Omega} \bar{\Gamma} q_{l f}^{f,-}
$$

Where $\bar{\Omega}$ and $\bar{\Gamma}$ can be calculated using Equations 3.4 and 3.5, respectively.

$$
\begin{gathered}
\bar{\Omega}=\left[\begin{array}{cc}
\cos \left(\frac{1}{2}\left\|\omega_{f}^{f}\right\| \Delta t\right) I_{3 \times 3}-[\psi \times] & \psi \\
-\psi^{T} & \cos \left(\frac{1}{2}\left\|\omega_{f}^{f}\right\| \Delta t\right)
\end{array}\right] \\
\bar{\Gamma}=\left[\begin{array}{cc}
\cos \left(\frac{1}{2}\left\|\omega_{l}^{l}\right\| \Delta t\right) I_{3 \times 3}-[\zeta \times] & -\zeta \\
\zeta^{T} & \cos \left(\frac{1}{2}\left\|\omega_{l}^{l}\right\| \Delta t\right)
\end{array}\right]
\end{gathered}
$$

Where $\psi$ and $\zeta$ are found in Equations 3.6 and 3.7, and $[x]$ is the notation used to represent the skew symmetric matrix of a vector.

$$
\begin{gathered}
\psi=\frac{\sin \left(\frac{1}{2}\left\|\omega_{f}^{f}\right\| \Delta t\right) \omega_{f}^{f}}{\left\|\omega_{f}^{f}\right\|} \\
\zeta=\frac{\sin \left(\frac{1}{2}\left\|\omega_{l}^{l}\right\| \Delta t\right) \omega_{l}^{l}}{\left\|\omega_{l}^{l}\right\|}
\end{gathered}
$$

\subsubsection{Required Attitude Transformation Mathematics}

The attitude propagation presented above represents attitude in quaternions. However, the values in the state vector are in radians and velocity propagation requires a rotation matrix. This section 
reviews the mathematics required to transform between the three representations [6].

\section{Quaternion to Transformation Matrix}

The components of the $3 \times 3$ rotation matrix $R$ are composed of elements of the quaternion representation of attitude as described in Equation 3.2.

$$
R=\left[\begin{array}{ccc}
q_{4}^{2}+q_{1}^{2}-q_{2}^{2}-q_{3}^{2} & 2\left(q_{1} q_{2}-q_{3} q_{4}\right) & 2\left(q_{1} q_{3}+q_{2} q_{4}\right) \\
2\left(q_{1} q_{2}+q_{3} q_{4}\right) & q_{4}^{2}-q_{1}^{2}+q_{2}^{2}-q_{3}^{2} & 2\left(q_{2} q_{3}-q_{1} q_{4}\right) \\
2\left(q_{1} q_{3}-q_{3} q_{4}\right) & 2\left(q_{2} q_{3}+q_{1} q_{0}\right) & q_{4}^{2}-q_{1}^{2}-q_{2}^{2}+q_{3}^{2}
\end{array}\right]
$$

\section{Transformation Matrix to Euler Angles}

Using the transformation $R$, the Euler angles can be found in radians using Equation 3.9. Where the first subscript relates to the row of the transformation matrix and the second the column. Where atan 2 is the four quadrant arctangent and is a standard MATLAB function.

$$
\begin{gathered}
\phi=\operatorname{atan} 2\left(R_{32}, R_{33}\right) \\
\theta=\operatorname{asin}\left(-R_{31}\right) \\
\psi=\operatorname{atan} 2\left(R_{21}, R_{11}\right)
\end{gathered}
$$

Noting that in order to convert the Euler representation from radians to degrees the values need only be multiplied by a scalar $\frac{180}{\pi}$.

\section{Euler Angles to Quaternion}

The Euler angles, in radians, can be converted back to a quaternion vector using Equation 3.10.

$$
\begin{aligned}
& q_{1}=\sin \left(\frac{\phi}{2}\right) \cos \left(\frac{\theta}{2}\right) \cos \left(\frac{\psi}{2}\right)-\cos \left(\frac{\phi}{2}\right) \sin \left(\frac{\theta}{2}\right) \sin \left(\frac{\psi}{2}\right) \\
& q_{2}=\cos \left(\frac{\phi}{2}\right) \sin \left(\frac{\theta}{2}\right) \cos \left(\frac{\psi}{2}\right)+\sin \left(\frac{\phi}{2}\right) \cos \left(\frac{\theta}{2}\right) \sin \left(\frac{\psi}{2}\right) \\
& q_{3}=\cos \left(\frac{\phi}{2}\right) \cos \left(\frac{\theta}{2}\right) \sin \left(\frac{\psi}{2}\right)-\sin \left(\frac{\phi}{2}\right) \sin \left(\frac{\theta}{2}\right) \cos \left(\frac{\psi}{2}\right) \\
& q_{4}=\cos \left(\frac{\phi}{2}\right) \cos \left(\frac{\theta}{2}\right) \cos \left(\frac{\psi}{2}\right)+\sin \left(\frac{\phi}{2}\right) \sin \left(\frac{\theta}{2}\right) \sin \left(\frac{\psi}{2}\right)
\end{aligned}
$$




\subsection{Relative Velocity Update}

The relative velocity formulation is taken from [16] and is updated by adding the previous relative velocity prediction to the relative velocity rate, or acceleration.

$$
v_{l f}^{f,+}=v_{l f}^{f,-}+\dot{v}_{l f}^{f,+} \Delta t
$$

Where, due to rotating nature of the resolving axis, $\dot{v}_{l f}^{f,+}$ is the summation of not only the applied force but two virtual forces that are generally refered to as the centrifugal and Coriolis forces, represnted by subscripts $c$ and $C$, respectively. These additional acceleration terms are generally associated with velocity updates in the local navigation frame where Earth's spin is the source of the rotation rate and accelerations in Equations 3.12 and 3.13 [6]. However, in this application the rotating reference frame is the body axis of the follower aircraft, thus the angular rates and accelerations in Equations 3.12 and 3.13 are of the follower aircraft's body frame.

$$
\begin{gathered}
\dot{v}_{c}=-\left(\dot{\omega}_{f} \times r_{l f}^{f,-}\right)-\left(\left[\omega_{f} \times\right]\left[\omega_{f} \times\right] r_{l f}^{f,-}\right) \\
\dot{v}_{C}=-2\left(\omega_{f} \times v_{l f}^{f,-}\right)
\end{gathered}
$$

Where $\dot{\omega}$ for the follower aircraft can be calculated at any time step, $t$, through the numerical differentiation shown in Equation 3.14, where $\Delta t$ is time between reported IMU data.

$$
\dot{\omega}_{t}=\frac{\Delta \theta_{t}-\Delta \theta_{t-1}}{\tau_{s}^{2}}
$$

Utilizing Equations 3.12 and 3.13, the relative velocity update equation can be expanded to 3.15 .

$$
v_{l f}^{f,+}=v_{l f}^{f,-}-\left(\dot{v}_{c}+\dot{v}_{C}+a_{f}-a_{l}+R_{E}^{f}\left(g_{f}^{E}-g_{l}^{E}\right)\right) \Delta t
$$

Through the assumption $\left(g_{f}^{E} \approx g_{l}^{E}\right)$ making the last term of 3.15 negligible. Additionally, $\Delta t$ can be distributed to each $a$ term simplifying the expression to Equation 3.16.

$$
v_{l f}^{f,+}=v_{l f}^{f,-}-\left(\dot{\omega}_{f} \times r_{l f}^{f,-}\right) \Delta t-\left(\left[\omega_{f} \times\right]\left[\omega_{f} \times\right] r_{l f}^{f,-}\right) \Delta t-2\left(\omega_{f} \times v_{l f}^{f,-}\right) \Delta t+\Delta v_{f}-R_{l}^{f} \Delta v_{l}
$$

Where $\Delta v$ represents incremental velocity changes measured in the indicated body frame and $R_{l}^{f}$ is 
the transformation matrix from the leader's body frame to the followers and can be calculated by aplying Euqation 3.8 to the quaternion found in Equation 3.3.

\subsection{Relative Position Update}

The simplest of the relative updates is the position state. Where the newly estimated velocity is used to estimate the incremental change in displacement since the previous time step. This position delta is then added to the previous estimation of position as shown in Equation 3.17.

$$
r_{l f}^{f,+}=r_{l f}^{f,-}+\left(v_{l f}^{f,+}\right) \Delta t
$$




\section{Chapter 4}

\section{Computer Vision Algorithm}

The algorithm presented in this chapter was developed and tested by Systems and Technology Research and is presented here as a supplement to the content of this thesis. Parts of this chapter are taken from the conference paper "Unmanned Aerial Vehicle Relative Navigation in GPS Denied Environments" [19]

The image matching technique presented in this chapter is once again, reliant on the proximity of the aircraft. Present or past images collected by the leader aircraft are matched to the most recent image collected by the camera on the follower platform. The result of the algorithm is a rotation matrix from the body axis of the leader aircraft to the follower and a unit vector that represents the direction from the leader to the follower in the follower's body frame. Figure 4.1 represents the process used and the resulting measurements.

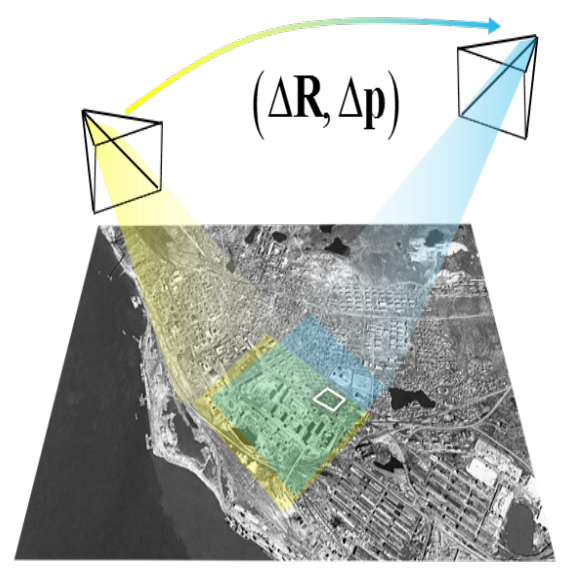

Figure 4.1: Computer Vision Algorithm Visual Representation

Computer vision updates provide a crucial directional component to the algorithm in this ap- 
plication. While rotation information gained from the measurement is helpful, it is not as valuable due to high rate magnetometer data providing attitude updates. However, the ranging radio measurements alone do not provide enough information to properly estimate position without the unit vector measurement provided by this computer vision algorithm. Systems and Technology Research (STR) provided the error model used in the simulation for computer vision unit vector and rotation measurements. The error distribution of these measurements where empirically found by testing the algorithm described in this chapter on several image data-sets. It should be noted that in this section references to cameras $A$ and $B$ are the downward facing cameras on the leader and follower aircraft, respectively.

A commonly used model of image formation is the pinhole camera model, which assumes that the model for a camera $p$ can be parameterized using 3 elements: a $3 \times 3$ upper triangular intrinsic camera parameter, $K^{p}$, a $3 \times 3$ rotation matrix, $R_{W}^{p}$, representing a rotation of $3 \mathrm{D}$ world points to camera-centered 3D points, and lastly a 3 element vector representing camera $p$ 's position in world coordinates, $c^{p}$. Given these parameters the full $3 \times 4$ perspective projective matrix can be defined by Equation 4.1 .

$$
P^{p}=K^{p}\left[R_{W}^{p}-R_{W}^{p} c^{p}\right]
$$

Then the transformation projecting a $3 \mathrm{D}$ world point $y$ onto $2 \mathrm{D}$ image point $x^{p}$ using the homogeneous coordinate representation can be defined by Equation 4.2.

$$
\tilde{x}^{p}=P^{p} \tilde{y}
$$

Supposing that two such camera models for cameras A and B were available the relative pose can be directly calculated by subtracting the positions and calculating the rotation matrix between camera $\mathrm{A}$ and $\mathrm{B}$.

$$
R_{A}^{B}=R_{W}^{B}\left(R_{W}^{A}\right)^{T}
$$

With the goal of associating pixels between two images and calculating the relative transformation a four step process is employed. First, a latent Dirichlet allocation (LDA) model is trained on an expected like set of imagery data pre-flight or in real time from the imagery of the leader platform [23]. This model uses vector quantinization in the 128 dimensional feature space provided by Scale Invariance Feature Transform (SIFT) [24] to form a discrete set of "words" (so named because the 
LDA model was developed for document analysis). From these words a set of "topics" is identified in the training imagery. The likelihood of a certain word appearing in a certain topic and the likelihood of a topic appearing in an image can then be established.

Next, the set of images is searched from the two on-board cameras to find corresponding images between aircraft using the LDA model and a Hidden Markov Model (HMM). Once again features are extracted from the imagery and are mapped to words. Corresponding images are found by using the statistical model to calculate the maximum likelihood match of the set of features from platform B to the models learned on the images of platform A. The HMM is used to enforce time consistency between observations. Figure 4.2 shows a plate notation diagram of the LDA process.

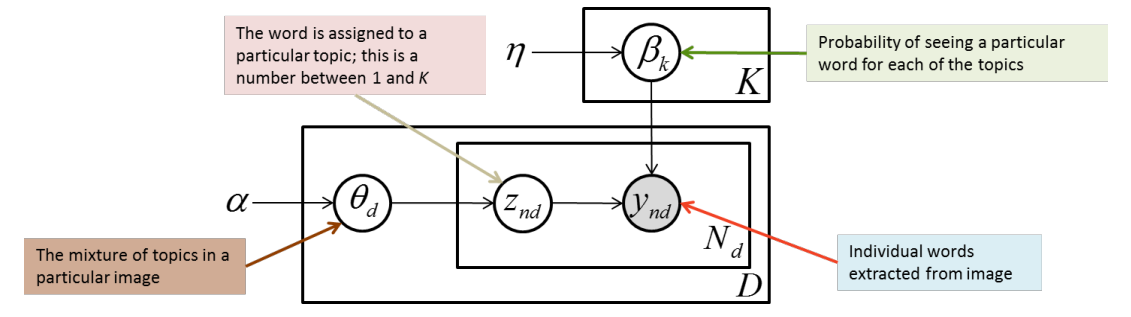

Figure 4.2: Latent Dirichlet Plate Notation Model

Then a frame-to-frame matching algortihm is used to align the top matching candidate images from the set of corresponding images. Lastly, given the set of correspondences between any two images and the $3 \times 3$ intrinsic camera parameter matrix, $\mathbf{K}$, the five-point algorithm [25] can be used to compute an estimate of the essential matrix.

$$
E=R_{A}^{B}\left[t_{A}^{B} \times\right]
$$

Where $R_{A}^{B}$ represents the relative rotation from platform A's coordinate frame to platform B's and $t_{A}^{B}$ represents the relative translation between the two coordinate frames. Thus, a 3D point from platform A's coordinate frame can be transformed into platform B's coordinate frame given the true relative pose.

$$
x^{B}=R_{A}^{B} x^{A}+t_{A}^{B}
$$

Since $E$ is being recovered from correspondences, only the direction of $t_{A}^{B}$ can be computed due to the projective ambiguity.

The relative pose from those correspondences is calculated using the five-point algorithm in a Random Sampling and Consensus (RANSAC) [26] loop for robustness. 


\section{Chapter 5}

\section{Measurement and Error Source}

\section{Models}

This chapter describes the methods of each of the on-board sensors, including the observation equations that map state estimates, the error distributions of the simulated sensor measurements, and expected differences of these error models in a physical application.

\subsection{Ultra-Wideband Ranging Radio}

UWB ranging radio measurements provide measurements of absolute distance between the platforms. The measurement model is the magnitude, or $L_{2}$ norm, of the estimated relative position.

$$
\left\|r_{l f}^{f}\right\|_{2}=\sqrt{\left(\hat{x}_{l f}^{f}\right)^{2}+\left(\hat{y}_{l f}^{f}\right)^{2}+\left(\hat{z}_{l f}^{f}\right)^{2}}
$$

The error distribution of UWB measurements in the simulation is a Gaussian distribution with $0 \mathrm{~cm}$ mean and $25 \mathrm{~cm}$ variance, $\mathcal{N}(0 \mathrm{~cm}, 25 \mathrm{~cm})$. As opposed to this simplified model experimental testing of UWB ranging radio shows that the error is non-Gaussian with signal drop-outs. Additionally, it has been found that the error is commonly multimodal.

\subsection{Tri-axial Magnetometers}

Both platforms' magnetometers provide measurements in each axis of the platform's body frame in units of nano Tesla $(n T)$. The measurements are used to compare the attitude of the leader platform 
with respect to the attitude of the follower. The measurement model is the mapping of the leader platform's magnetometer readings to the body frame of the follower platform shown in Equation 5.2. Where $R_{l}^{f}$ is the rotation matrix from the leader's body frame to the follower's body frame constructed from the the relative state attitude estimates.

$$
M_{f}=R_{l}^{f} M_{l}
$$

The magnetometer error distribution is Gaussian with 0 mean and $525 \mathrm{nT}$ variance, $\mathcal{N}(0 n T, 525 n T)$. Conversely, physical magnetometer sensor values would experience hard and soft iron distortion values, as well as other natural and man-made disturbances not present in the magnetic field earth model used to generate the simulated data [27].

\subsection{Computer Vision}

This section addresses the two elements of data acquired from computer vision algorithms conducted on the video imagery provided by each platforms downward facing camera. The error distributions displayed for these two measurement updates where provided by STR. Since these measurements errors are non-Gaussian, the standard deviation used in the measurement noise matrix for each of these computer vision components was found by over bounding the empirical distributions, so that the uncertainty of measurements was increased. This method was applied to prevent outlying measurements from being trusted too much at the expense of trusting accurate measurements less. The model used in this simulation has no time dependence, meaning that outliers occur at random with no regard to the previous computer vision estimates. This is an inaccurate assumption, as an outlier at one time step through computer vision analysis would be more likely to cause outliers in the following measurements as well. Additionally, the computer vision model is invariant to the altitude of either of the aircraft. Practical application of the algorithm would show correlation between the relative altitude of the aircraft in the ability to match images, as well as the overall altitude of an individual aircraft regarding the diversity of features found in the on-board imagery. Finally, as alluded to in Chapter 4, in order for the algorithm to be applied there must be over lapping segments of imagery from the follower aircraft to the archived images from the leader aircraft, meaning the ability of the algorithm to find an accurate solution is dependent on the flight path's of the involved UAVs. 


\subsubsection{Rotation Element}

The rotation elements provided by the computer vision algorithm are reported in Euler angles, and represent the rotation from the follower platform to the leader. As such, the computer vision measurements are directly compared to the current relative attitude estimation.

$$
C V_{\text {Euler }}=\boldsymbol{\theta}_{l f}^{f}
$$

The error model used provides a rotation matrix to represent the error. This rotation matrix is multiplied by the truth relative Euler angles to provide a simulated computer vision attitude update. The error distribution is determined by multiplying a random $3 \times 1$ vector by the rotation matrix and subtracting the resulting vector from the original. The distribution is visualized in Figure 5.1 and the statistics of the distribution are in Table 5.1.

\section{Error Distribution of Rotation Error}

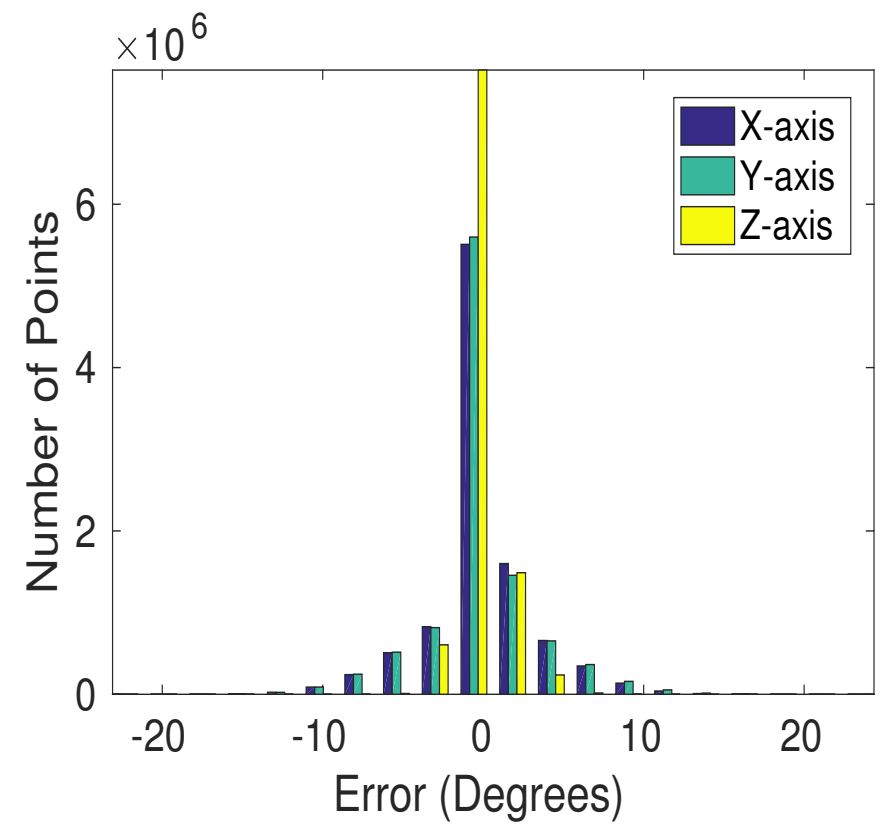

Figure 5.1: Distribution of Computer Vision Rotation Error

\begin{tabular}{|c|c|c|c|c|}
\hline Axis & Mean & Standard Deviation & Skewness & Kurtosis \\
\hline X-axis & $-0.0309^{\circ}$ & $3.1706^{\circ}$ & -0.0788 & 6.0555 \\
\hline Y-axis & $0.0189^{\circ}$ & $3.2518^{\circ}$ & 0.0894 & 6.0417 \\
\hline Z-axis & $0.0531^{\circ}$ & $1.2002^{\circ}$ & 0.6486 & 6.7281 \\
\hline
\end{tabular}

Table 5.1: Statistics of Distribution for Rotational Component of Computer Vision Measurements 


\subsubsection{Unit Vector Element}

The directional measurement provided by the computer vision update is a unit vector pointing from the follower aircraft to the leader aircraft. The model to map the state vector to the measurement is to form a unit vector from the relative position estimate.

$$
C V_{\text {unit }}=\frac{1}{\left\|r_{l f}^{f}\right\|_{2}}\left[\begin{array}{c}
x_{l f}^{f} \\
y_{l f}^{f} \\
z_{l f}^{f}
\end{array}\right]
$$

The error model provided is an additive error vector for the unit vector calculated from the true relative position values. The distribution of the error for each axis can be seen in Figure 5.2 and the statistics of the distribution are displayed in Table 5.2.

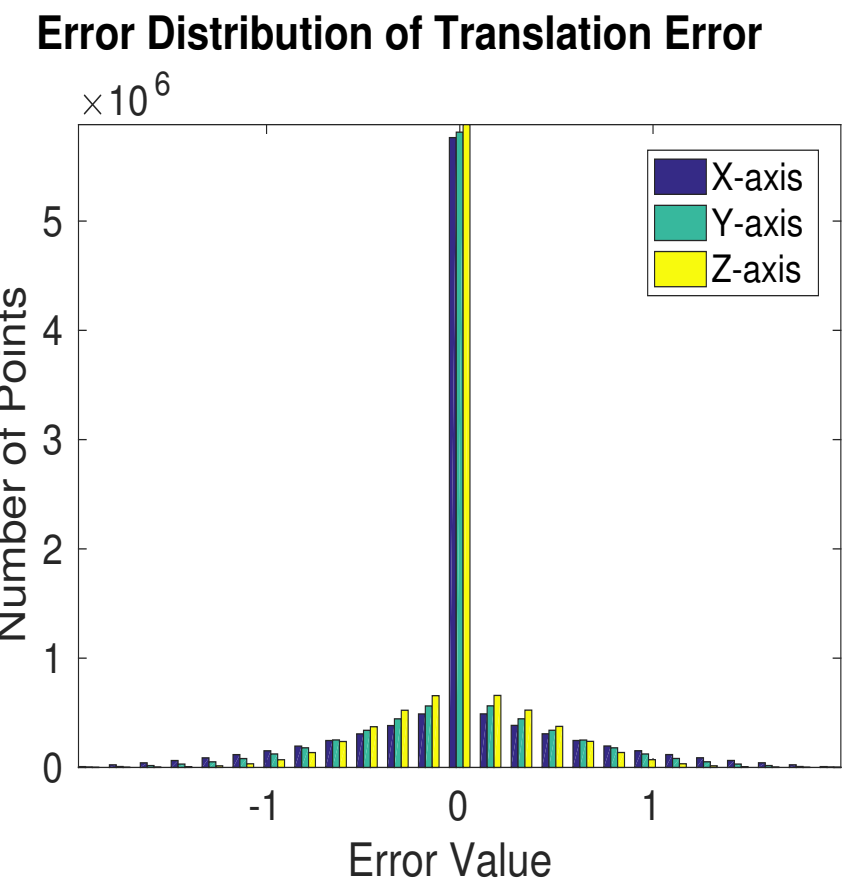

Figure 5.2: Distribution of Computer Vision Unit Vector Error

\begin{tabular}{|c|c|c|c|c|}
\hline Axis & Mean & Standard Deviation & Skewness & Kurtosis \\
\hline X-axis & 0.00016 & 0.47293 & -0.00048 & 6.23130 \\
\hline Y-axis & 0.00008 & 0.39861 & 0.00188 & 6.82021 \\
\hline Z-axis & -0.00002 & 0.31304 & 0.00033 & 6.75993 \\
\hline
\end{tabular}

Table 5.2: Statistics of Distribution for Unit Vector Component of Computer Vision Measurements 


\section{Chapter 6}

\section{Unscented Kalman Filter}

The Kalman filter [28] is an optimal linear state space estimator that has been adopted by the navigation community as an effective way to fuse multiple data sources. The processes contains two recursive steps: propagation of state based on a system model and a state update based on sensor measurements. In many navigation applications, including the work in this thesis, the model used to propagate the state is the output of the INS, while update measurements are provided by any one of many on-board sensors, most commonly GNSS. However, the standard Kalman filter is only optimized for linear propagation and update models. There are two common methods to adjust the linear Kalman filter to non-linear application. The Extended Kalman Filter (EKF) uses similar methodology to the linear Kalman filter but linearizes around the current state estimate [29]. The Unscented Kalman Filter (UKF), which is what the rest of this thesis will focus on, utilizes the unscented transformation [30] instead of performing the linearization required in the EKF. The UKF selects a specific set of sample points, which will be referred to as sigma-points, as inputs to the non-linear system and captures the posterior mean and covariance after the transformation. It has been documented computationally that for most non-linear application the unscented transformation provides more accurate mean and variance estimates and thus has been selected for use in this thesis $[31],[32],[33],[34],[35]$.

\subsection{Unscented Transform}

The selection of sigma-points differs from a Monte Carlo particle filter in the sense that the points are not selected at random, but are instead calculated using a deterministic algorithm which is

described in Equation 6.1. Where the resulting $\chi_{k-1}$ is a $[L \times(2 L+1)]$ matrix, with $L$ being the 
number of elements in the state vector.

$$
\chi_{k-1}=\left[\begin{array}{lll}
\hat{x}_{k-1} & \hat{x}_{k-1}+\sqrt{(L+\lambda)} \sqrt{P_{k-1}} & \hat{x}_{k-1}-\sqrt{(L+\lambda)} \sqrt{P_{k-1}}
\end{array}\right]
$$

The parameter $\lambda$ in Equation 6.1 is calculated using Equation 6.2. Where $\alpha$ is a scaling parameter that determines the spread of sigma-points and $\kappa$ is parameter that can be used to tune the higher order moments of the approximations [36].

$$
\lambda=\alpha^{2}(L+\kappa)-L
$$

\subsection{Prediction Transformation}

The prediction step of the algorithm uses the sigma-points calculated from the previous state $\hat{\chi}_{k-1}$ and passed each column, $i$, through the non-linear relative inertial navigation equations and storing the transformed points, $\hat{x}_{k \mid k-1}$, as seen in Equation 6.3. Subscript $k \mid k-1$ is read to mean values of time step $k$ given values from time step $k-1$.

$$
\chi_{k \mid k-1}^{(i)}=f\left(\chi_{k-1}^{(i)}\right), i=0,1, \ldots 2 L
$$

The post transformation mean, $\hat{x}_{k \mid k-1}$, and covariance, $P_{k \mid k-1}$, are then calculated using Equations 6.4 and 6.5 .

$$
\begin{gathered}
\hat{x}_{k \mid k-1}=\sum_{i=0}^{2 L} \eta_{i}^{m} \chi_{k \mid k-1}^{(i)} \\
P_{k \mid k-1}=Q+\sum_{i=0}^{2 L} \eta_{i}^{c}\left(\chi_{k \mid k-1}^{(i)}-\hat{x}_{k \mid k-1}\right)\left(\chi_{k \mid k-1}^{(i)}-\hat{x}_{k \mid k-1}\right)^{T}
\end{gathered}
$$

Where the weighting parameters $\eta_{i}^{m}$ and $\eta_{i}^{c}$ are calculated in Equation 6.6 using previously established values and an additional scaling parameter $\beta$ which includes information about the prior distribution. For Gaussian processes this parameter is optimally set at 2 [36].

$$
\begin{gathered}
\left.\eta_{0}^{m}=\frac{\lambda}{(L+\lambda}\right) \\
\left.\eta_{0}^{c}=\frac{\lambda}{(L+\lambda}\right)+1-\alpha^{2}+\beta \\
\eta_{i}^{m}=\frac{1}{2(L+\lambda)} \\
\eta_{i}^{c}=\eta_{i}^{c}
\end{gathered}
$$




\subsection{Observation Transformation}

Similar to process for the prediction step, the measurement update step begins by passing the calculated sigma-points of the current state, $\chi_{k \mid k-1}$, through the non-linear observation models.

$$
\psi_{k \mid k-1}^{(i)}=h\left(\chi_{k \mid k-1}^{(i)}\right)
$$

Equation 6.7 transforms each sigma-point vector to the units and scale of the measurement data $z_{k}$, resulting in a $\left[n_{z} \times 2 L+1\right]$ output sigma-matrix $\psi_{k \mid k-1}^{(i)}$. Where $n_{z}$ is number of sensor measurements available at time step k. These output sigma-points are then used to calculate the predicted output, output covariance, and the cross-covariance between the state and output as seen in Equations $6.8-6.10$.

$$
\begin{gathered}
\hat{z}_{k \mid k-1}=\sum_{i=0}^{2 L} \eta_{i}^{m} \psi_{k \mid k-1}^{(i)} \\
P_{k}^{y y}=R_{k}+\sum_{i+0}^{2 L} \eta_{i}^{c}\left(\psi_{k \mid k-1}^{(i)}-\hat{z}_{k \mid k-1}\right)\left(\psi_{k \mid k-1}^{(i)}-\hat{z}_{k \mid k-1}\right)^{T} \\
P_{k}^{x y}=\sum_{i+0}^{2 L} \eta_{i}^{c}\left(\chi_{k \mid k-1}^{(i)}-\hat{x}_{k \mid k-1}\right)\left(\psi_{k \mid k-1}^{(i)}-\hat{z}_{k \mid k-1}\right)^{T}
\end{gathered}
$$

\subsection{Measurement Update}

The measurement update step uses the previously calculated output covariance, state-output cross covariance, output prediction to update the predicted state vector based on the collected measurements. The Kalman gain which is optimized to minimize mean squared error is calculated in Equation 6.11.

$$
K_{k}=P_{k}^{x y}\left(P_{k}^{y y}\right)^{-1}
$$

The new state estimate can then be calculated using Equation 6.12 where $z_{k}$ is the vector containing sensor measurements.

$$
\hat{x}_{k}=\hat{x}_{k \mid k-1}+K_{k}\left(z_{k}-\hat{z}_{k \mid k-1}\right)
$$

The difference of the sensor measurement and mapped state, $z_{k}-\hat{z}_{k \mid k-1}$ is known as the innovation 
residual and is commonly considered when evaluating the quality of provided measurements and the tuning of matrices described in the next section. The update error covariance matrix is then found using Equation 6.13.

$$
P_{k}=P_{k \mid k-1}-K_{k} P_{k}^{y y} K_{k}^{T}
$$

Being a recursive algorithm, the entire process is then repeated for the next time step, $k+1$.

\subsection{Assigning Process and Measurement Noise}

The Kalman filter has three tuning matricies that must be specified for the specific application. Since the Kalman filter is known to be sensitive to tuning parameters [37], this section will detail the specific tuning for this application.

\section{State Covariance Matrix - P}

The initial state covariance matrix, $P_{o}$ is used to define the amount of uncertainty that is assumed in the initial states. In this application, $P_{p o s}, P_{v e l}$, and $P_{a t t}$, are the amount of uncertainty in the position, velocity, and attitude states at the initialization of the filter. The initial error values are assigned a normal distribution with mean and standard deviation in the simulation environment. Therefore, $P_{\text {pos,vel,att }}$ becomes a $3 \times 3$ diagonal matrix with $\sigma_{\text {pos,vel,att }}^{2}$ components.

$$
P_{0}=\left[\begin{array}{ccc}
P_{\text {pos }} & 0_{3 \times 3} & 0_{3 \times 3} \\
0_{3 \times 3} & P_{\text {vel }} & 0_{3 \times 3} \\
0_{3 \times 3} & 0_{3 \times 3} & P_{a t t}
\end{array}\right]
$$

\section{Process Noise Covariance Matrix - Q}

The process noise covariance matrix, $Q$, describes how much the state is expected to vary over the interval of one time step due to the uncertainty in the dynamic model used to propagate the state. In this case the model is the relative inertial equations. Thus, $Q_{v e l}$ and $Q_{a t t}$ are related to the velocity random walk (VRW) and angular random walk (ARW) associated with the IMU used, respectively. In a typical one platform application the VRW or ARW associated with each axis of the aircraft would be treated as a standard deviation and simply squared. However, with multiple aircraft as a part of the solution a different approach must be taken, where the follower aircraft process noise matrix is added to the leader aircraft process noise matrix rotated into the follower's body frame 
using the general rotation formulation in Equation 6.15. Where $R_{l}^{f}$ is the rotation from the leader to the follower frame and $Q_{l}$ represents $Q_{p o s}, Q_{v e l}$, or $Q_{a t t}$ for the leader aircraft.

$$
Q_{l}^{f}=R_{l}^{f} Q_{l} R_{l}^{f T}
$$

When $Q_{l}$ is a scalar diagonal matrix, this rotation has no effect. In this application VRW is equal for every axis meaning that $Q_{v e l}$, from one of the identical aircraft is simply doubled. However, ARW has a different value in the roll axis of the IMU, meaning that the rotation shown in Equation 6.15 when applied to the leaders $Q_{a t t}$ will result in a fully populated $[3 \times 3]$ matrix.

$$
\begin{aligned}
& Q_{v e l}=2\left[\begin{array}{ccc}
V R W^{2} & 0 & 0 \\
0 & V R W^{2} & 0 \\
0 & 0 & V R W^{2}
\end{array}\right] \\
& Q_{a t t}=\left[\begin{array}{ccc}
A R W_{f_{x}}^{2} & 0 & 0 \\
0 & A R W_{f_{y}}^{2} & 0 \\
0 & 0 & A R W_{f_{z}}^{2}
\end{array}\right]+R_{l}^{f}\left[\begin{array}{ccc}
A R W_{l_{x}}^{2} & 0 & 0 \\
0 & A R W_{l_{y}}^{2} & 0 \\
0 & 0 & A R W_{l_{z}}^{2}
\end{array}\right] R_{l}^{f T}
\end{aligned}
$$

For this application the standard deviation of position in each axis was estimated to be 0.1 meter acquired from the compounding uncertainty in velocity and attitude estimation. Once again, due to the dual platform system the leader and follower aircraft must be summed to calculate the final $Q_{p o s}$ of the system. The rotation from the leader to follower from Equation 6.15 will have no effect on the scalar matrix, once again resulting in the multiplication of a scalar 2 as seen in Equation 6.18.

$$
Q_{\text {pos }}=2\left[\begin{array}{ccc}
0.1^{2} & 0 & 0 \\
0 & 0.1^{2} & 0 \\
0 & 0 & 0.1^{2}
\end{array}\right]
$$

The final full process noise matrix $Q$ then becomes:

$$
Q=\left[\begin{array}{ccc}
Q_{\text {pos }} & 0_{3 \times 3} & 0_{3 \times 3} \\
0_{3 \times 3} & Q_{v e l} & 0_{3 \times 3} \\
0_{3 \times 3} & 0_{3 \times 3} & Q_{a t t}
\end{array}\right]
$$




\section{Measurement Noise Covariance Matrix - R}

The measurement noise covariance matrix, $R$, is used to account for the uncertainty in the sensor readings included in the measurement update step. The size of this matrix will vary based on which measurements are available at each discrete time step. For simplicity the $R$ matrix for each measurement will be reviewed separately. While the ranging radio, $r r$, element in this application is a scalar, the remaining elements in the matrix are $3 \times 3$ diagonal matrix constructed from the sigma values outlined in the Measurement Models and Sensor Error chapter of this thesis.

$$
R_{r r}=\sigma_{r r}^{2}
$$

Similar to components of the process noise matrix the magnetometer, mag, elements in the measurement noise matrix require a scalar 2 product, because readings from two identical but independent magnetometers are used in the system to calculate the innovation residual.

$$
R_{m a g}=2\left[\begin{array}{ccc}
\sigma_{x}^{2} & 0 & 0 \\
0 & \sigma_{y}^{2} & 0 \\
0 & 0 & \sigma_{z}^{2}
\end{array}\right]
$$

Only one generalized $R_{C V}$ matrix is displayed for the ration, $C V r$, and translation, $C V t$, components as both computer vision measurement components use the same general matrix structure, where $\sigma_{x, y, z}$ are the empirical standard deviation values described in the Measurement Models and Sensor Error chapter of this thesis.

$$
R_{C V}=\left[\begin{array}{ccc}
\sigma_{x}^{2} & 0 & 0 \\
0 & \sigma_{y}^{2} & 0 \\
0 & 0 & \sigma_{z}^{2}
\end{array}\right]
$$

For applications in which the unit vector measurements are more concentrated around the true value a covariance model which accounts for dependencies of a unit vector could be considered [38].

$$
R_{3 \times 3}=\sigma_{\phi}\left[I_{3 \times 3}-b b^{T}\right]
$$

Where $b$, is the unit vector measurement and $\sigma_{\phi}$ is a scalar representing the pointing error of the unit vector in radians. However, these methods proved to be impractical for this error model as the range of measurement error was too large. 
The block diagonal measurement noise matrix found in Equation 6.24 is the matrix used in the UKF when all measurements are available at a discrete time step $k$.

$$
R_{k}=\left[\begin{array}{cccc}
R_{r r} & 0_{1 \times 3} & 0_{1 \times 3} & 0_{1 \times 3} \\
0_{3 \times 1} & R_{m a g} & 0_{3 \times 3} & 0_{3 \times 3} \\
0_{3 \times 1} & 0_{3 \times 3} & R_{C V r} & 0_{3 \times 3} \\
0_{3 \times 1} & 0_{3 \times 3} & 0_{3 \times 3} & R_{C V t}
\end{array}\right]
$$




\section{Chapter 7}

\section{Simulation and Monte Carlo}

The MATLAB simulation environment used was created at WVU [39] and makes used of the SatNav Toolbox [40]. The flight paths were generated using a Simulink aircraft model designed to resemble the dynamics of WVU's fixed wing UAV known as "Phastball", and was built during the university's work on formation flight of fixed wing UAVs [41],[17].

\subsection{Monte Carlo Design}

Monte Carlo analysis uses repeated random sampling of inputs to obtain an numerical distribution of the systems's output empirically. The random inputs in this analysis are the frequency of the on-board sensors, the quality of the IMUs, the flight path selected, scaling factors of magnetometer and ranging radio sensors, and the initial error present in the first state estimate. The sensitivity analysis consisted of 500 individual run of the algorithm where Tables $7.7-7.1$ show the distribution of simulated flights for several of the characteristics mentioned. Additionally, Table 7.8 shows the Gaussian distributions of initalization error in each axis of each state, where it is assumed the initial state is found using cooperative methods such as [18].

\begin{tabular}{|c|c|}
\hline Quality & Number of Trials \\
\hline Automotive & 112 \\
\hline Tactical & 125 \\
\hline Intermediate & 128 \\
\hline Aviation & 135 \\
\hline
\end{tabular}

Table 7.1: Distribution of IMU Quality into Monte Carlo Analysis 


\begin{tabular}{|c|c|}
\hline Flight Path & Number of Trials \\
\hline 1 & 201 \\
\hline 2 & 187 \\
\hline 3 & 112 \\
\hline
\end{tabular}

Table 7.2: Distribution of Flight Paths into Monte Carlo Analysis

\begin{tabular}{|c|c|}
\hline Frequency $(\mathrm{Hz})$ & Number of Trials \\
\hline 5 & 124 \\
\hline 10 & 108 \\
\hline 25 & 131 \\
\hline 50 & 137 \\
\hline
\end{tabular}

Table 7.3: Distribution of Ranging Radio Frequencies into Monte Carlo Analysis

\begin{tabular}{|c|c|}
\hline Frequency (Hz) & Number of Trials \\
\hline$<1$ & 104 \\
\hline $1-2$ & 88 \\
\hline $2-3$ & 104 \\
\hline $3-4$ & 105 \\
\hline $4-5$ & 99 \\
\hline
\end{tabular}

Table 7.4: Distribution of Ranging Radio Error Scale Factors into Monte Carlo Analysis

\begin{tabular}{|c|c|}
\hline Frequency $(\mathrm{Hz})$ & Number of Trials \\
\hline 10 & 93 \\
\hline 25 & 110 \\
\hline 50 & 146 \\
\hline 100 & 151 \\
\hline
\end{tabular}

Table 7.5: Distribution of Magnetometer Frequencies into Monte Carlo Analysis 


\begin{tabular}{|c|c|}
\hline Frequency (Hz) & Number of Trials \\
\hline$<1$ & 121 \\
\hline $1-2$ & 108 \\
\hline $2-3$ & 104 \\
\hline $3-4$ & 84 \\
\hline $4-5$ & 83 \\
\hline
\end{tabular}

Table 7.6: Distribution of Magnetometer Error Scale Factors into Monte Carlo Analysis

\begin{tabular}{|c|c|}
\hline Frequency $(\mathrm{Hz})$ & Number of Trials \\
\hline 1 & 127 \\
\hline 2 & 138 \\
\hline 5 & 117 \\
\hline 10 & 118 \\
\hline
\end{tabular}

Table 7.7: Distribution of Computer Vision Frequencies into Monte Carlo Analysis

\begin{tabular}{|l|c|c|}
\hline State & Mean & Standard Deviation \\
\hline Position & $0(\mathrm{~m})$ & $6(\mathrm{~m})$ \\
\hline Velocity & $0(\mathrm{~m} / \mathrm{s})$ & $3(\mathrm{~m} / \mathrm{s})$ \\
\hline Attitude & $0(\mathrm{deg})$ & $2(\mathrm{deg})$ \\
\hline
\end{tabular}

Table 7.8: Distribution of Initial Error for Each State

\subsection{Monte Carlo Results}

The error values presented in the Cumulative Distribution Functions (CDF) presented in this section were found by selecting the median of the Root Sum Squared (RSS) of position and attitude error at each discrete time step in each simulation.

Figure 7.1 is a CDF that includes every estimated epoch from all 500 simulated flights of the Monte Carlo analysis, and Table 7.9 provides precise median error values for the Monte Carlo flights. 

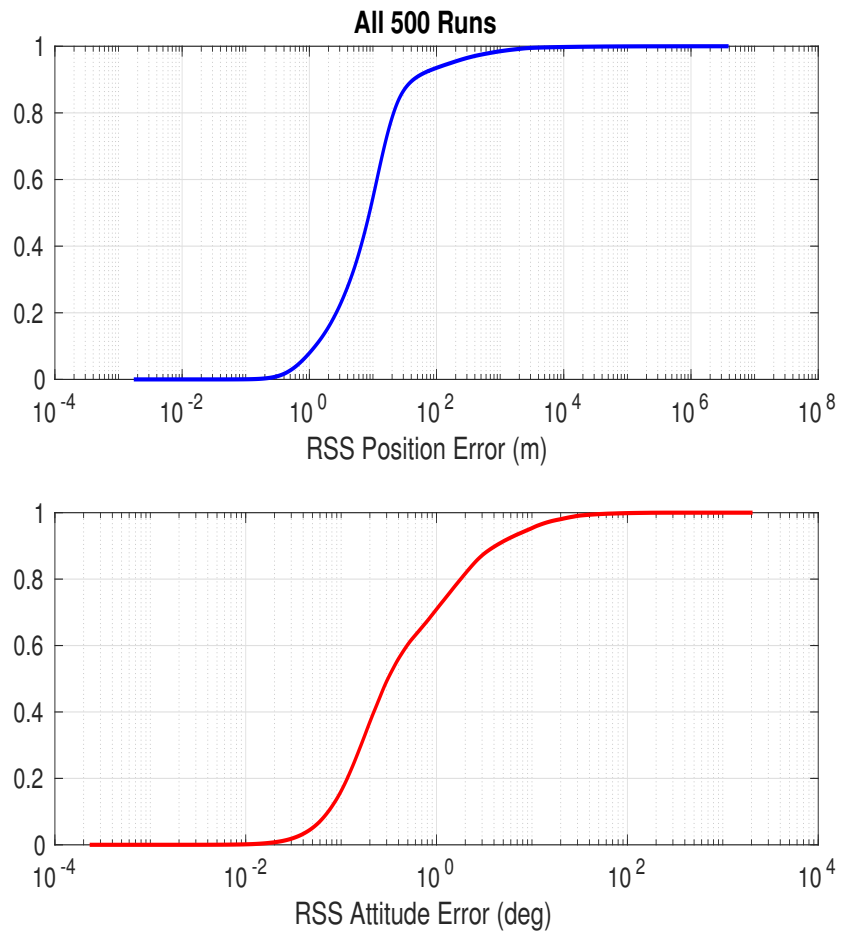

Figure 7.1: CDF for All Simulated Flights

\begin{tabular}{|c|c|}
\hline Median RSS Position Error & Median RSS Attitude Error \\
\hline 8.81 meters & $0.31^{\circ}$ \\
\hline
\end{tabular}

Table 7.9: Median RSS Error Values of All Monte Carlo Flights

Investigation of CDFs of IMU quality in Figures 7.2 and 7.3 shows large deviation between each grade of IMU, with a particularly large difference in error between the Automotive grade and the Tactical grade IMU. 

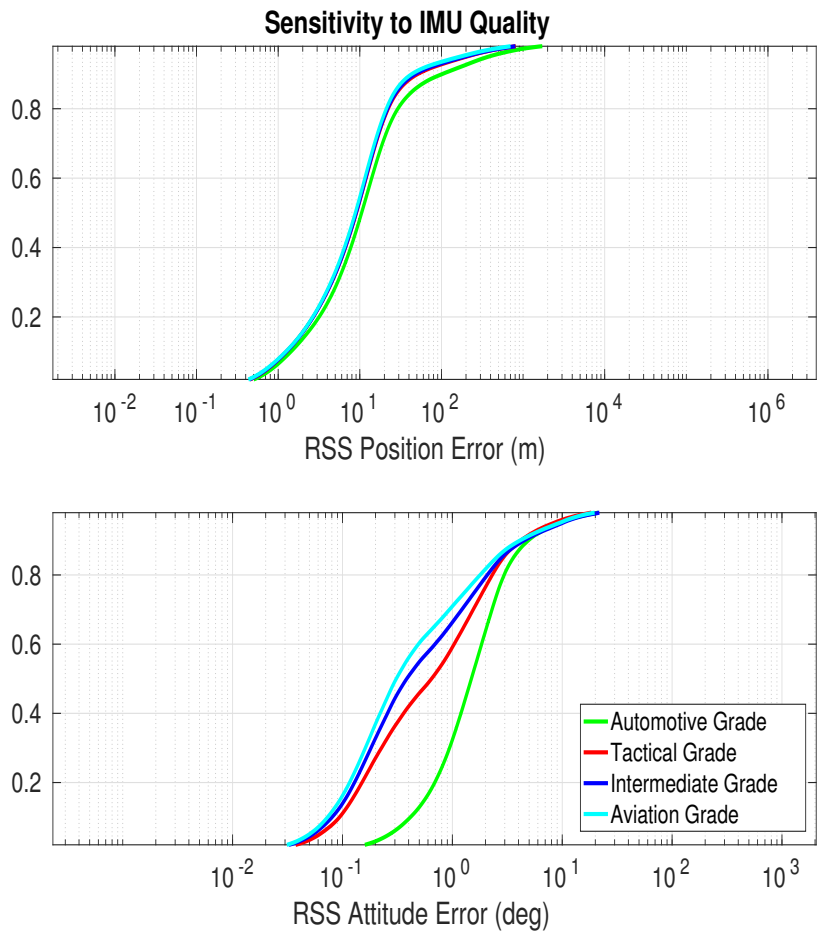

Figure 7.2: CDF for Inertial Measurement Unit Quality
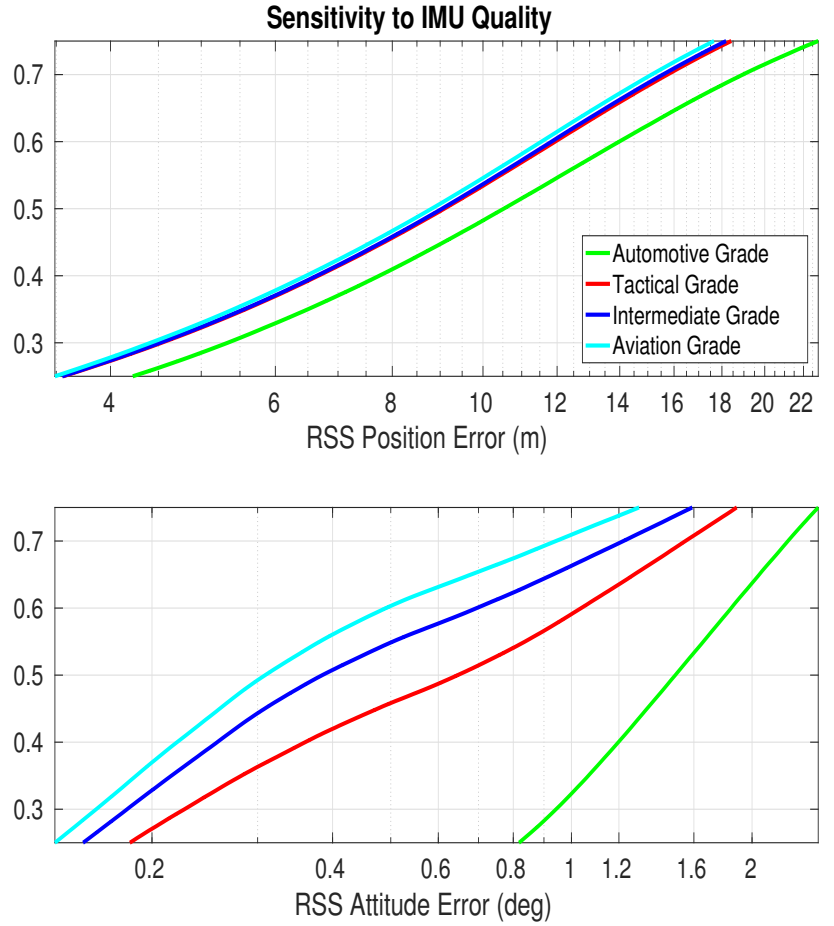

Figure 7.3: CDF for Inertial Measurement Unit Quality 
The flight paths tested in this study show little deviation at median error values in Figure 7.5. However, the values at the right tail of Figure 7.4 begins to show deviation, indicating that the bad estimates at larger ranges provided larger error, while good estimates where invariant to the total distance between the aircraft. Attitude error median values between flight paths 1 and 2 where nearly the same, while the most dynamic flight, flight path 3, resulted in larger RSS attitude error.
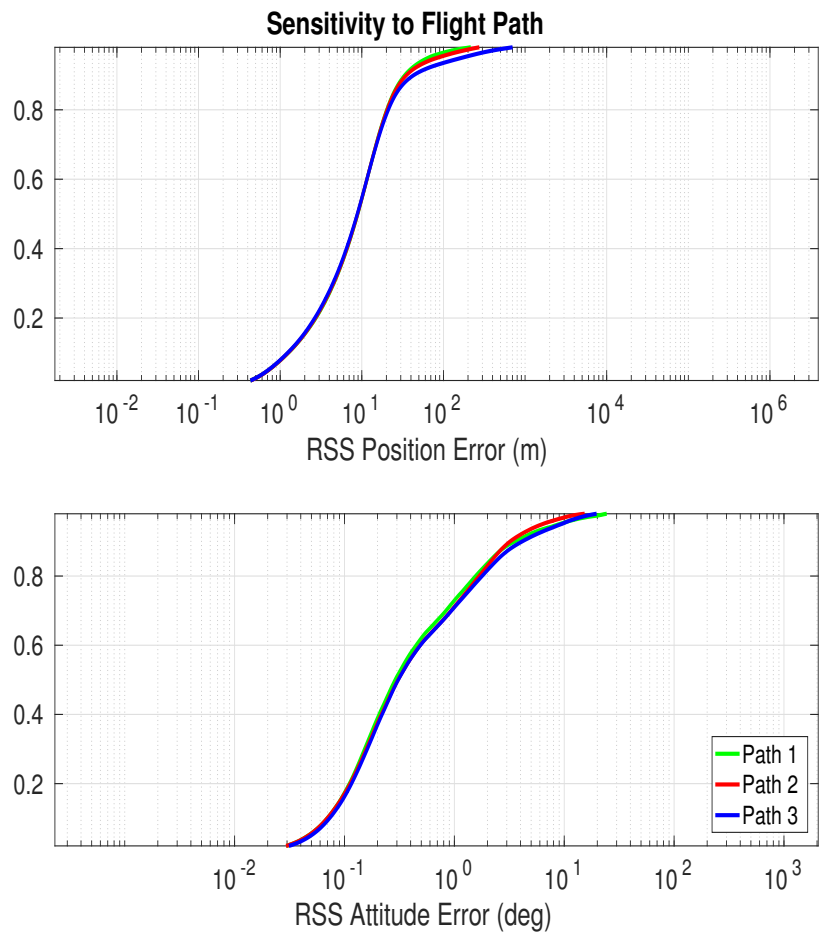

Figure 7.4: CDF for Flight Path Selections 

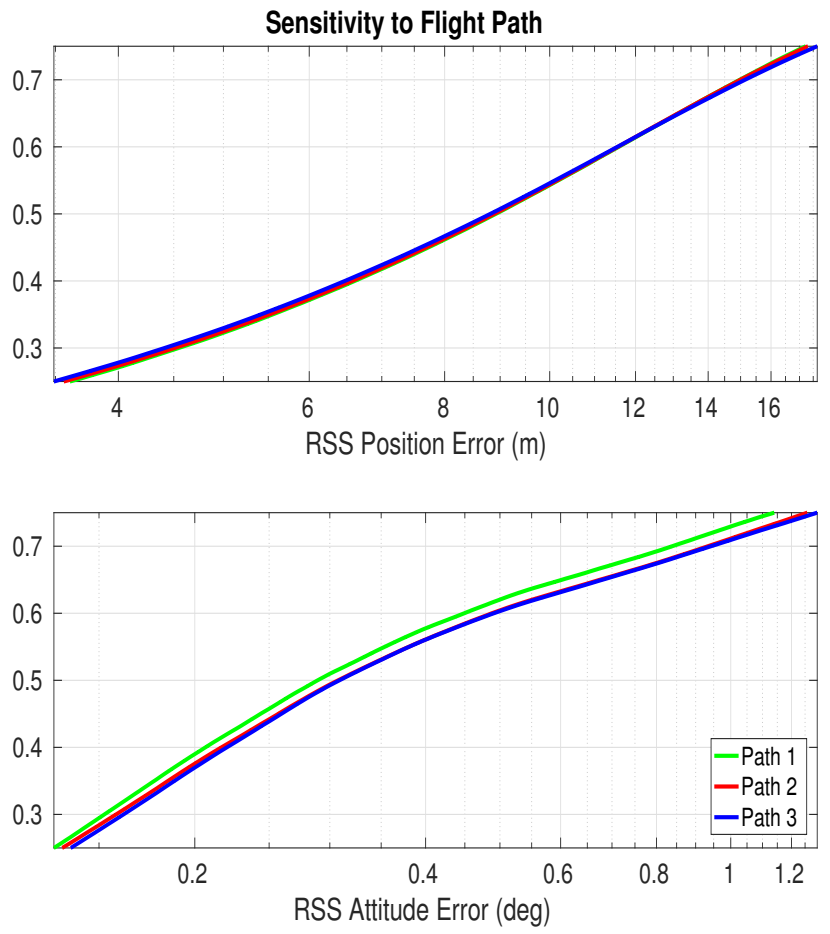

Figure 7.5: CDF for Flight Path Selections

As expected, the ranging radio update rate has no apparent effect on the attitude error. The positioning error of the tested frequencies displayed in Figures 7.6 and 7.7 shows a clear trend in the positioning accuracy as a function of update rate, where more update provide more accurate estimates. 

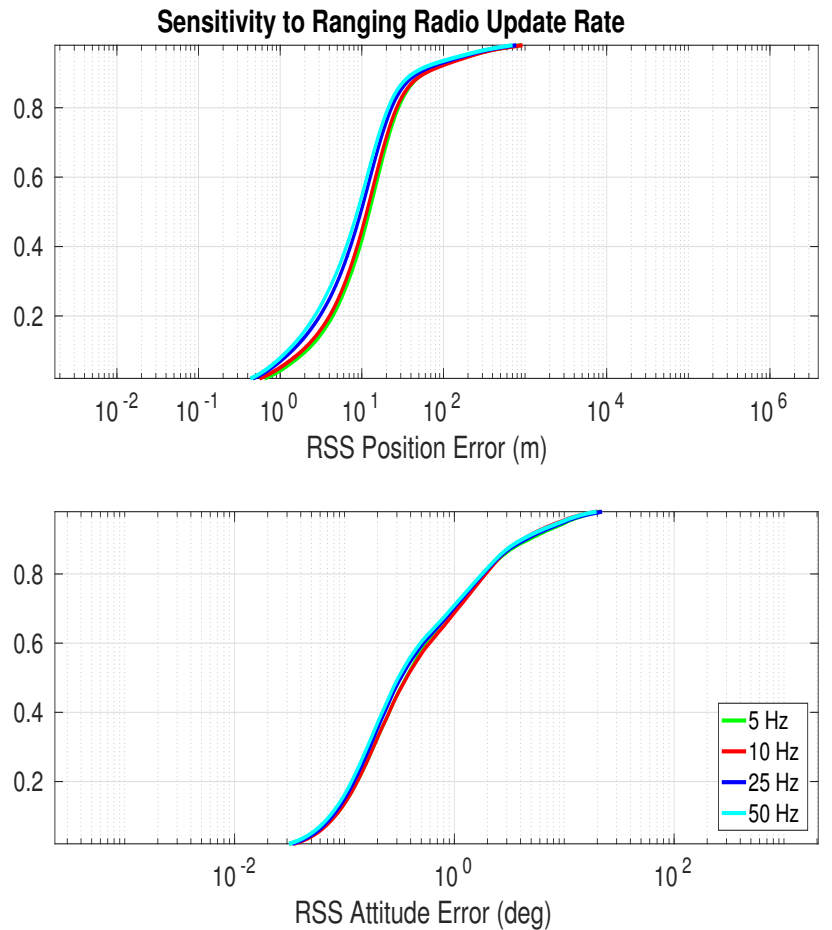

Figure 7.6: CDF for UWB Ranging Radio Measurement Frequency
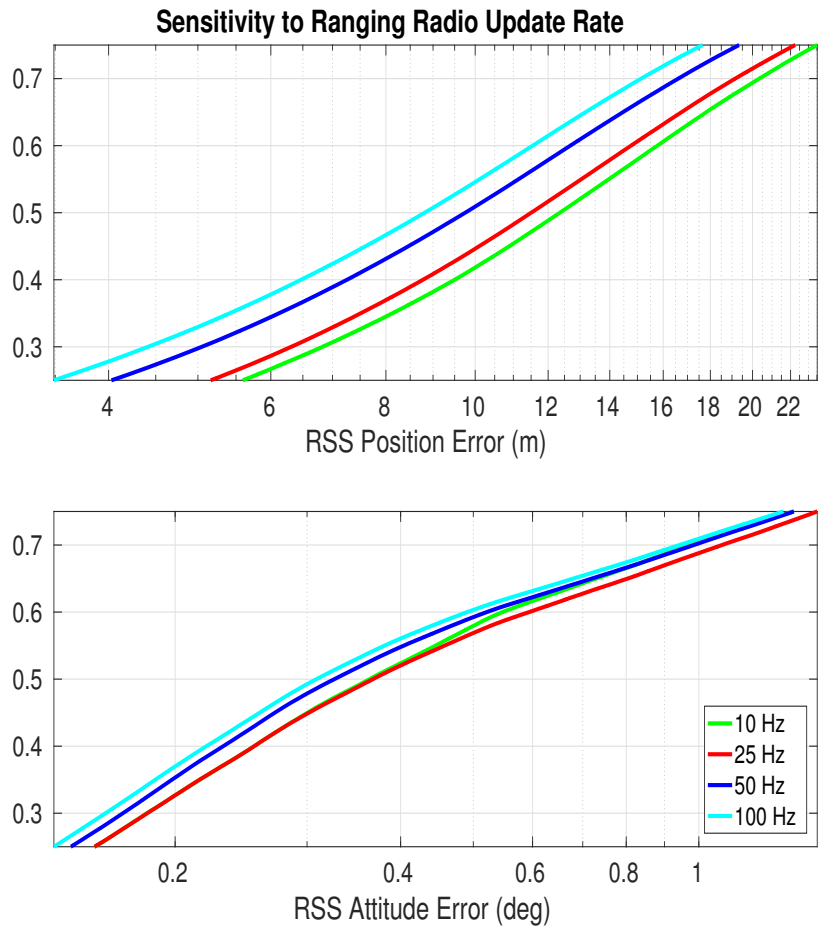

Figure 7.7: CDF for UWB Ranging Radio Measurement Frequency 
The scaling factors applied to the UWB measurements, Figures 7.8 and 7.9, appears to have no effect on the position indicating that the frequency at which measurements were provided had a larger impact on the solution than the quality of the measurements inside the scope of this study.
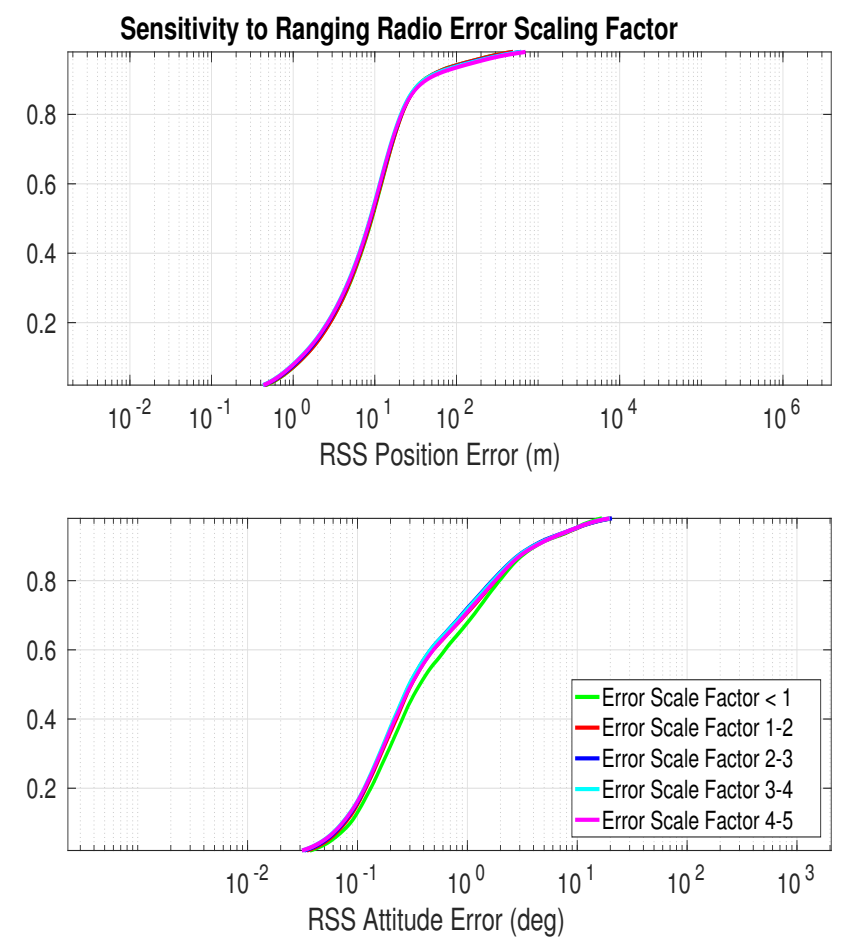

Figure 7.8: CDF for UWB Ranging Radio Measurement Error Scale Factor 

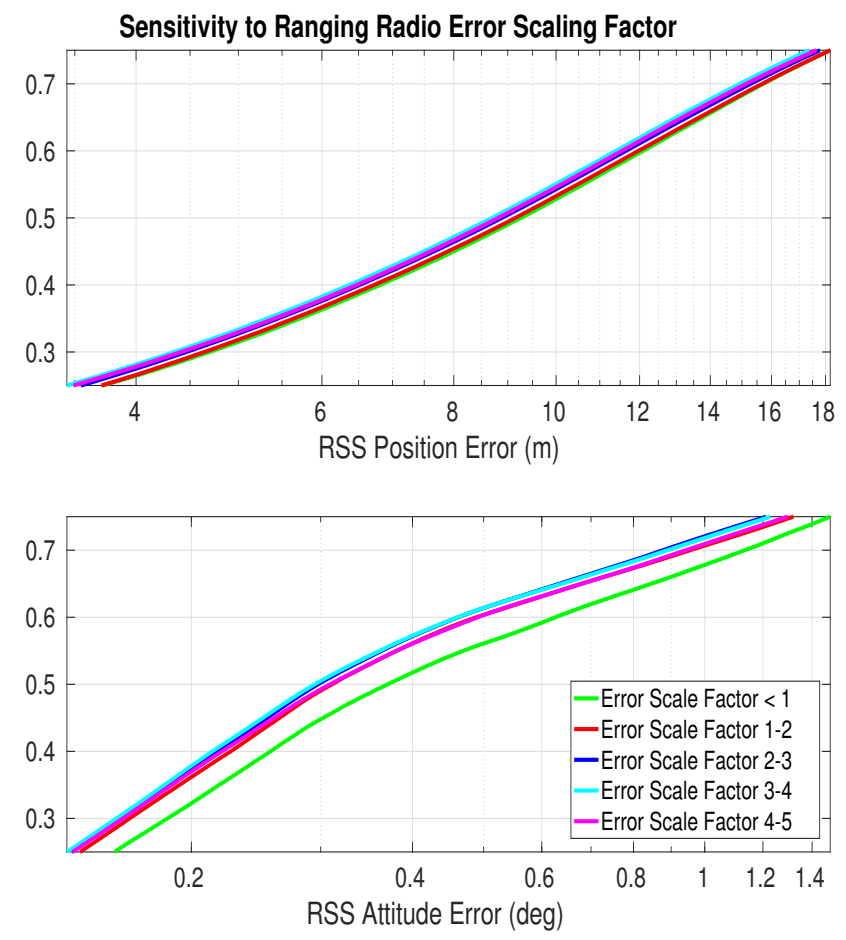

Figure 7.9: CDF for UWB Ranging Radio Measurement Error Scale Factor

Magnetometer update rate CDFs in Figures 7.10 and 7.11 show trends in both position and attitude estimate error, with higher update rates providing more accurate results. However, there is relatively little difference in error between 50 and $100 \mathrm{~Hz}$, indicating that the benefits of increasing the rate of magnetometer updates past $50 \mathrm{~Hz}$ for real application should be balanced with computational and finical factors. 

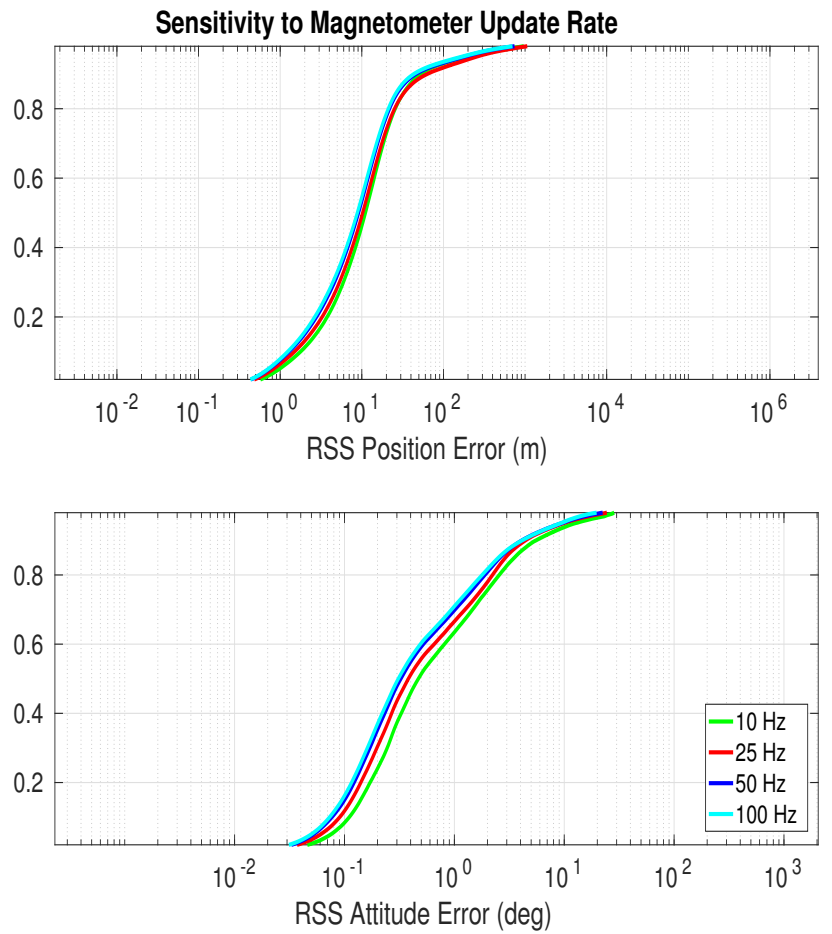

Figure 7.10: CDF for Magnetometer Measurement Frequency
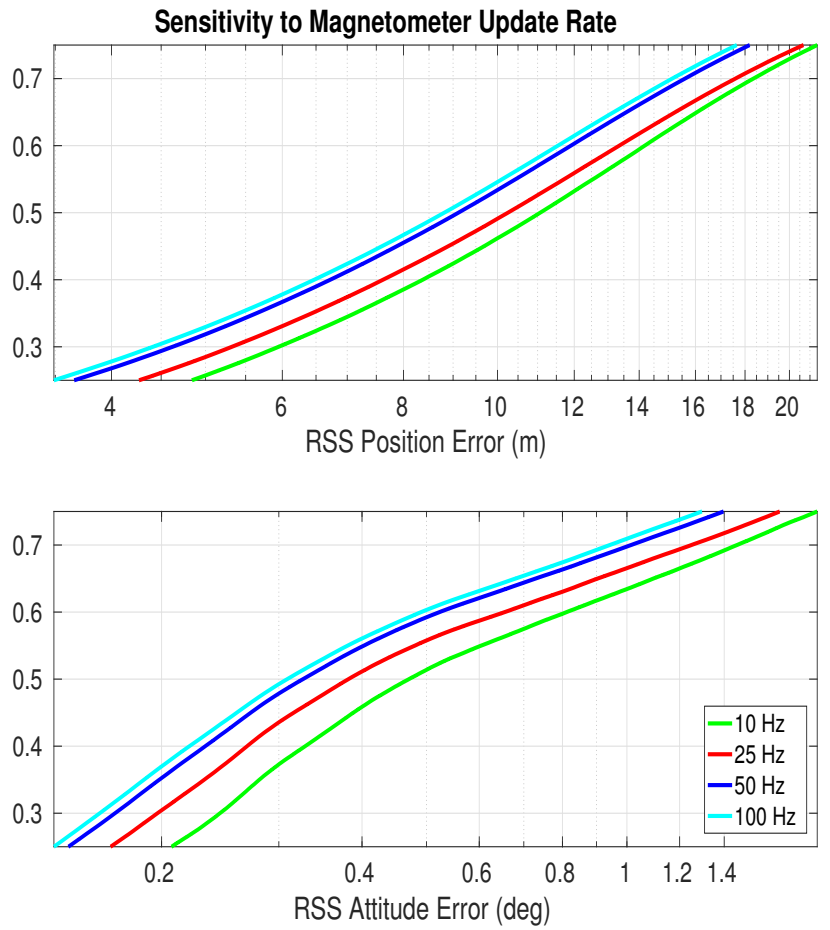

Figure 7.11: CDF for Magnetometer Measurement Frequency 
The magnetometer scaling factor CDFs shown in Figures 7.12 and 7.13 display trends in position error, with larger scaling factors resulting in larger error in estimates. The attitude solution also shows a trend among scaling factors between 1 and 5, which indicates that the poor results from the $<1$ scale factor simulations were likely dominated by other error sources.
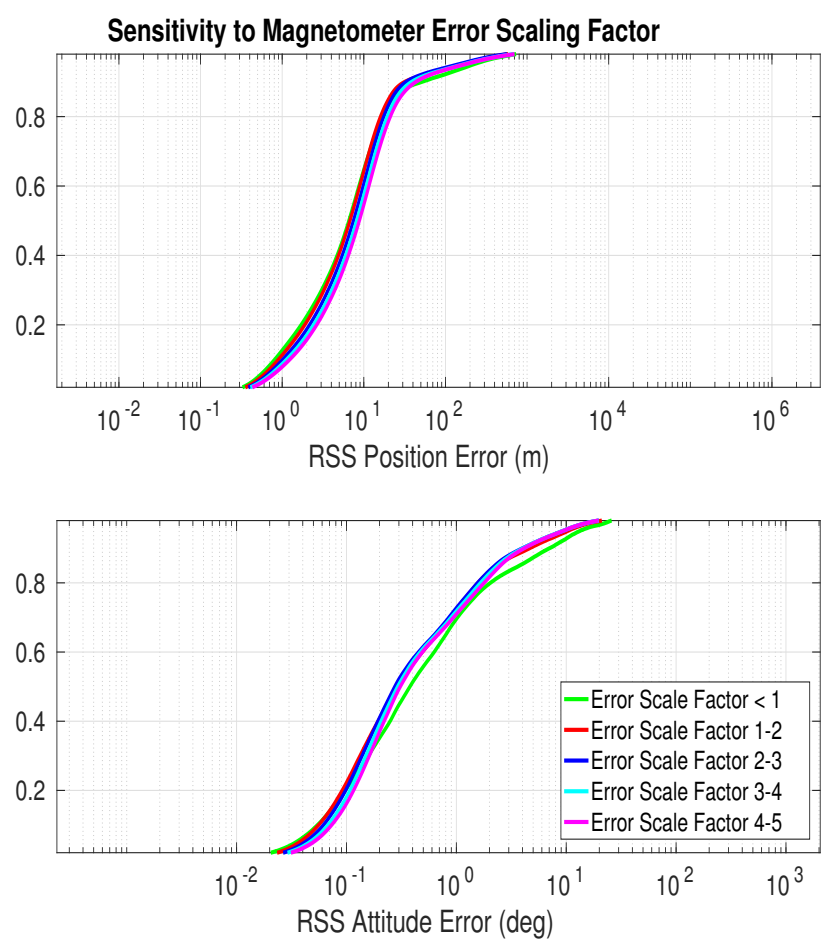

Figure 7.12: CDF for Magnetometer Measurement Error Scale Factor 

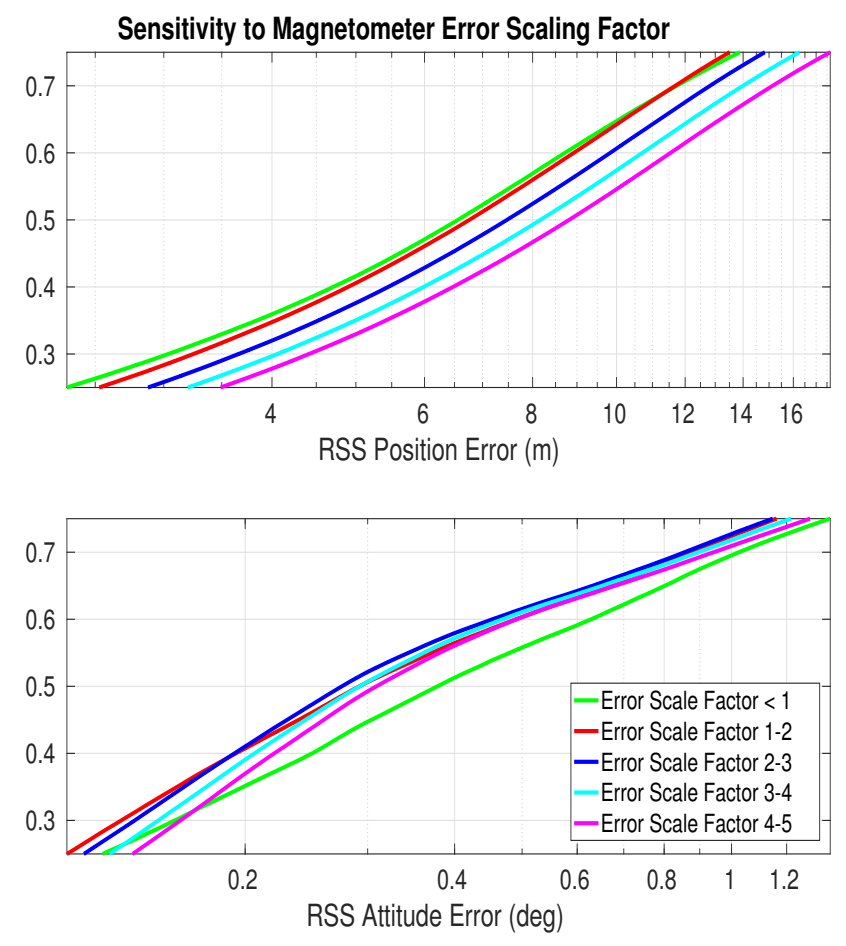

Figure 7.13: CDF for Magnetometer Measurement Error Scale Factor

The CDFs in Figures 7.14 and 7.15 shows that varying computer vision frequency produced little difference in attitude errors, while a distinguished trend in positioning error can be observed. This is an indication that the unit vector component of the computer vision measurements has more effect on the solution than the relative attitude measurement, which can be expected as magnetometer readings also provide attitude information at generally higher rates, while the only position update provided outside of computer vision updates is the ranging radio measurements which only provide a magnitude of relative position. 

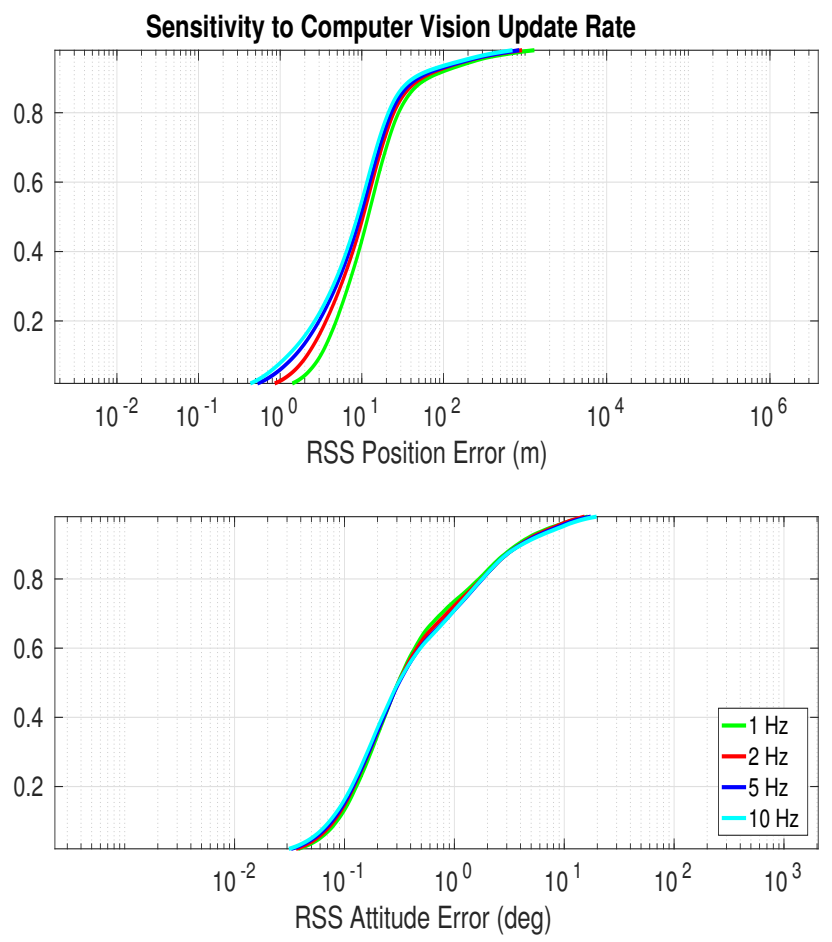

Figure 7.14: CDF for Computer Vision Measurement Frequency
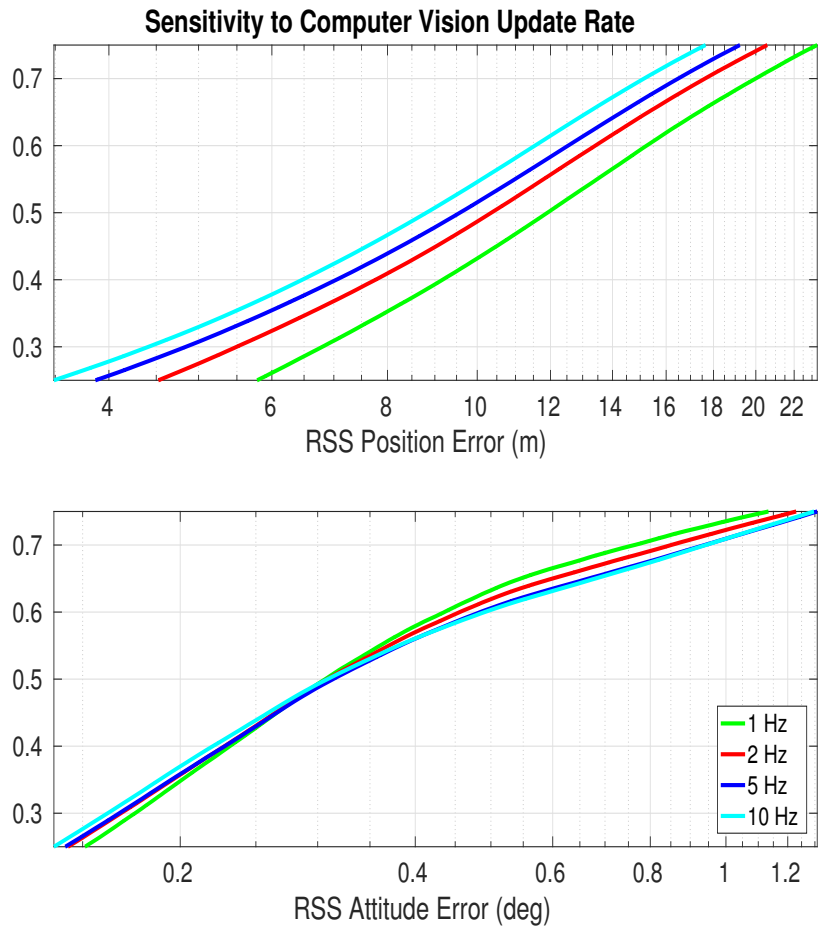

Figure 7.15: CDF for Computer Vision Measurement Frequency 


\subsection{Single Simulation Results}

Using the sensitivity analysis and technology constraints as a guide, a simulation was conducted with no error scaling values assigned to ranging radio or magnetometer measurements, as well as a perfect initial condition to analyze the performance of the algorithm across one run with ideal conditions.

\begin{tabular}{|c|c|}
\hline Measurement & Relavent Characteristic \\
\hline IMU & Intermediate Grade \\
\hline Flight Path & 2 \\
\hline Magnetometer & $100 \mathrm{~Hz}$ \\
\hline Ranging Radio & $10 \mathrm{~Hz}$ \\
\hline Computer Vision & $2 \mathrm{~Hz}$ \\
\hline
\end{tabular}

Table 7.10: Mean RSS Error Values at Several Percentages of Monte Carlo Flights

Figure 7.16 - 7.18 show a clear advantage in the performance of the UKF compared to an IMU only solution in both position and velocity, with the IMU drift creating significant error in both states in a matter of 50 seconds. With that said, it is also shown that in that same time frame the relative attitude of the IMU only solution is comparable to the UKF solution, both of which closely follow the trends of the truth value for the flight. 


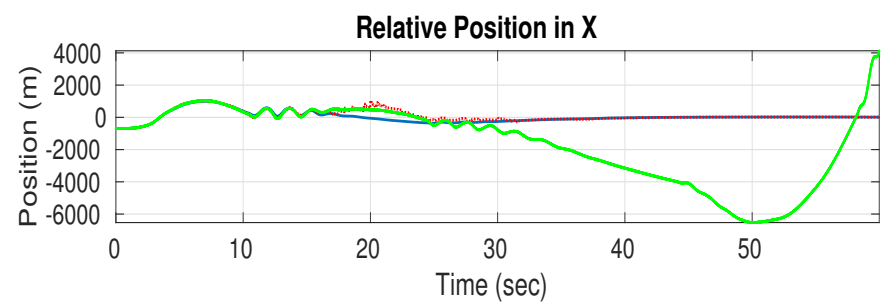

Relative Position in Y

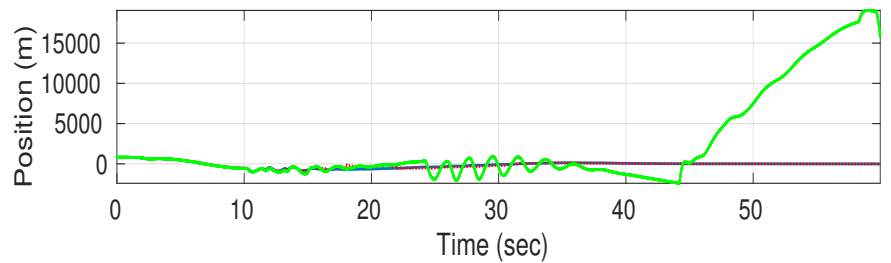

Relative Position in z

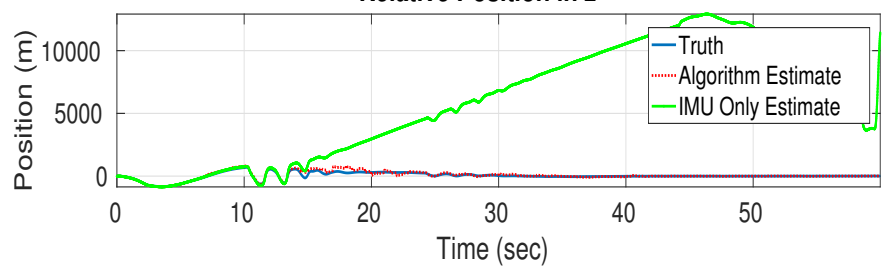

Figure 7.16: Comparison of Navigation Algorithm and IMU Only for Position Estimate


Figure 7.17: Comparison of Navigation Algorithm and IMU Only for Velocity Estimate 


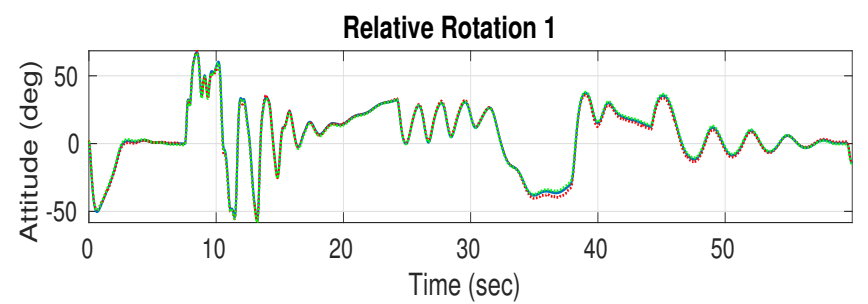

Relative Rotation 2

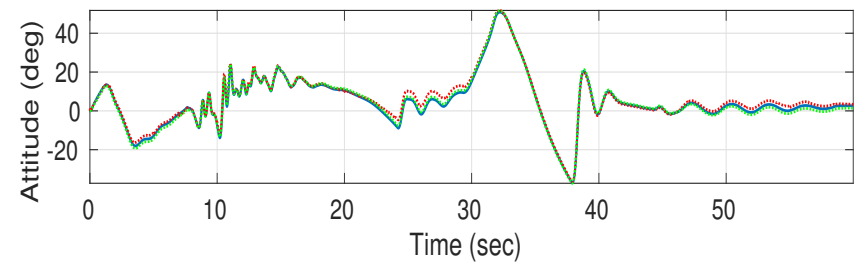

Relative Rotation 3

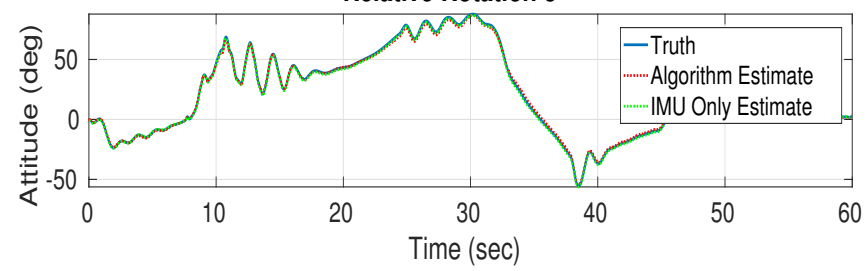

Figure 7.18: Comparison of Navigation Algorithm and IMU Only for Attitude Estimate

Figure 7.19 shows that it is only in the last 30 seconds of the simulated 8 minute flight that the the UKF solution proves advantageous as the drift of the IMU only solution noticeably begins to deviate from truth. At this same point in the flight the IMU solution for position results in over 1 million meters of error. 

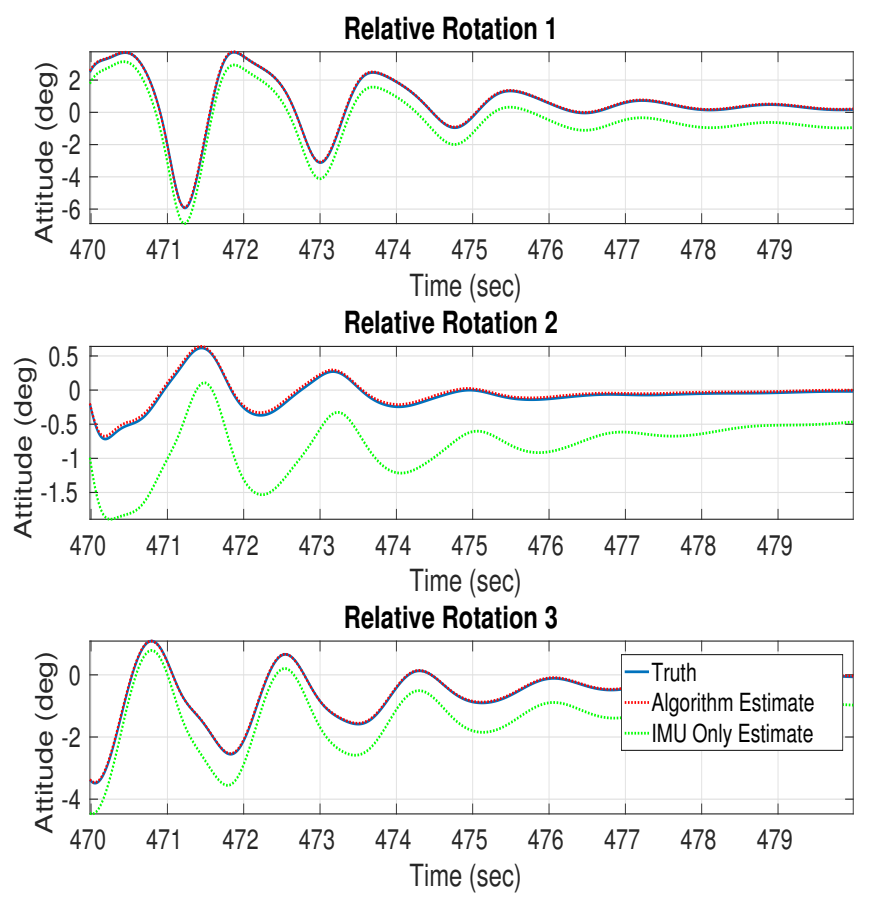

Figure 7.19: Comparison of Navigation Algorithm and IMU Only for Attitude Estimate

Figure 7.20 shows the UKF position solution in each axis compared to the truth in the first 50 seconds of the flight. As the flight progresses in time and the algorithm begins to rely on computer vision and ranging radio to correct IMU drift the solution deviates from truth. The large jumps seen around the 20 second mark in this portion of the flight are due to outliers in computer vision unit vector measurements that translate to large positioning errors when the magnitude of range is large. As seen in Figure 7.21 later in the flight these jumps are still present, however as the aircraft converge the errors in these unit measurements translate to smaller errors in position. Although the positioning solution is still noisy due to these outliers in unit vector measurements the mean of the UKF position solutions does center around the truth position. 

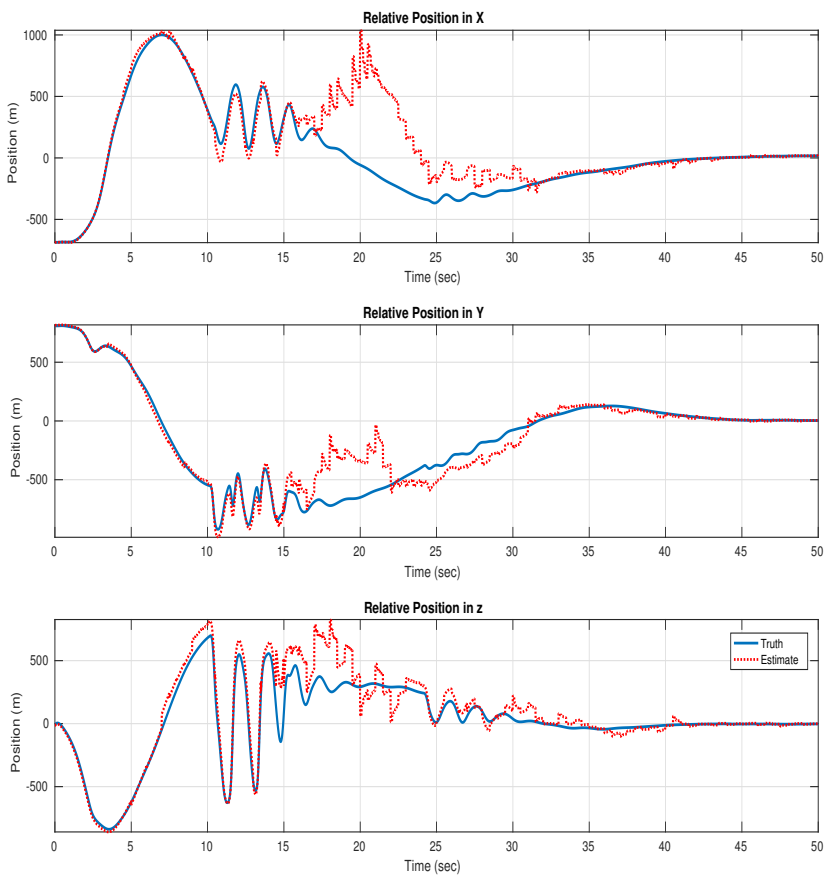

Figure 7.20: UKF Solution Compared to Truth in Beginning of Flight
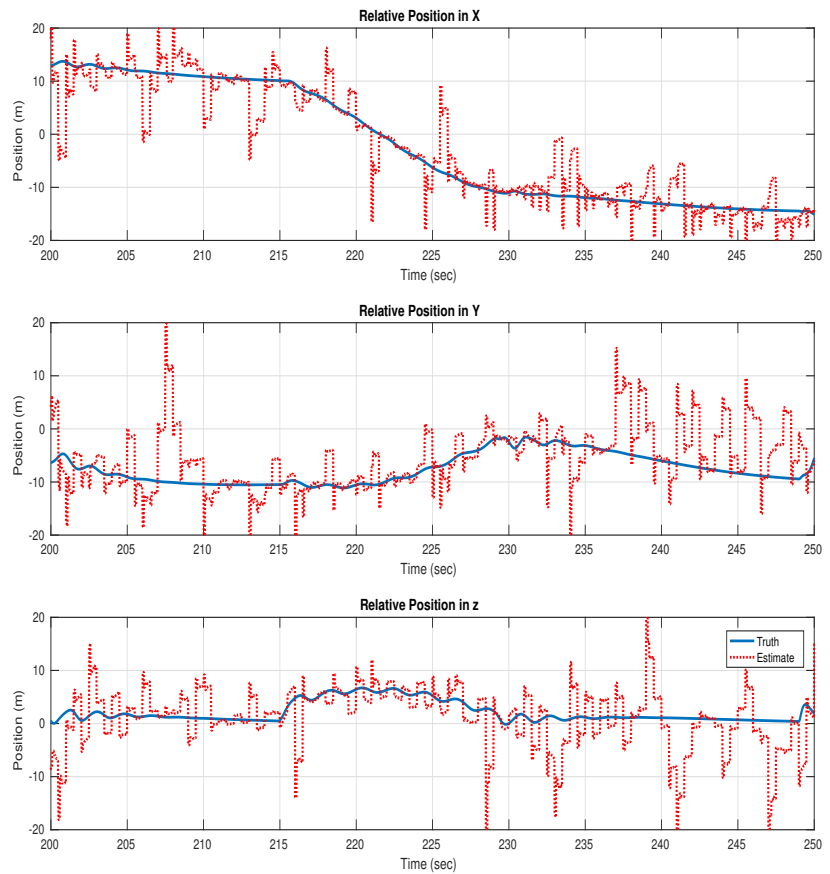

Figure 7.21: UKF Solution Compared to Truth after Aircraft Convergence 


\section{Chapter 8}

\section{Experimental Platform}

In addition to the study conducted in the simulation environment, an experimental set-up was designed with the intent of testing the navigation algorithm on data collected by a physical system. This chapter outlines the payload design of the two platforms and their capabilities with regards to future testing.

\subsection{Rover}

The ground rover was designed to be used as one of the moving platforms required to test the accuracy of the UKF in a real environment. The rover is equiped with an Analog Devices 16485AMLZ IMU [42], a Time Domain PulsON P-410 UWB ranging radio [43], and a NovAtel [44] GPS reciever with NovAtel Pinwheel antenna [45]. These sensors are interfaced using a Netburner MOD54415 [46], and an interface board specifically designed for this application at WVU by Scott Harper. Data collected from the IMU and UWB on-board the rover is collected via Netburner firmware and stored on a SD card on the Netburner board. Additionally, Pulse Per Second (PPS) data is taken from the GPS receiver breakout board and stored on the same SD card along with the Netburner's internal counter for time tag alignment purposes. The collected raw GPS data is stored on SD cards located on the GPS receiver's breakout board. The rover is also equiped with a single board computer that is used to interface with an Xbee modem [47] and speed controllers to control the rover's movement via keyboard input from a nearby laptop. 


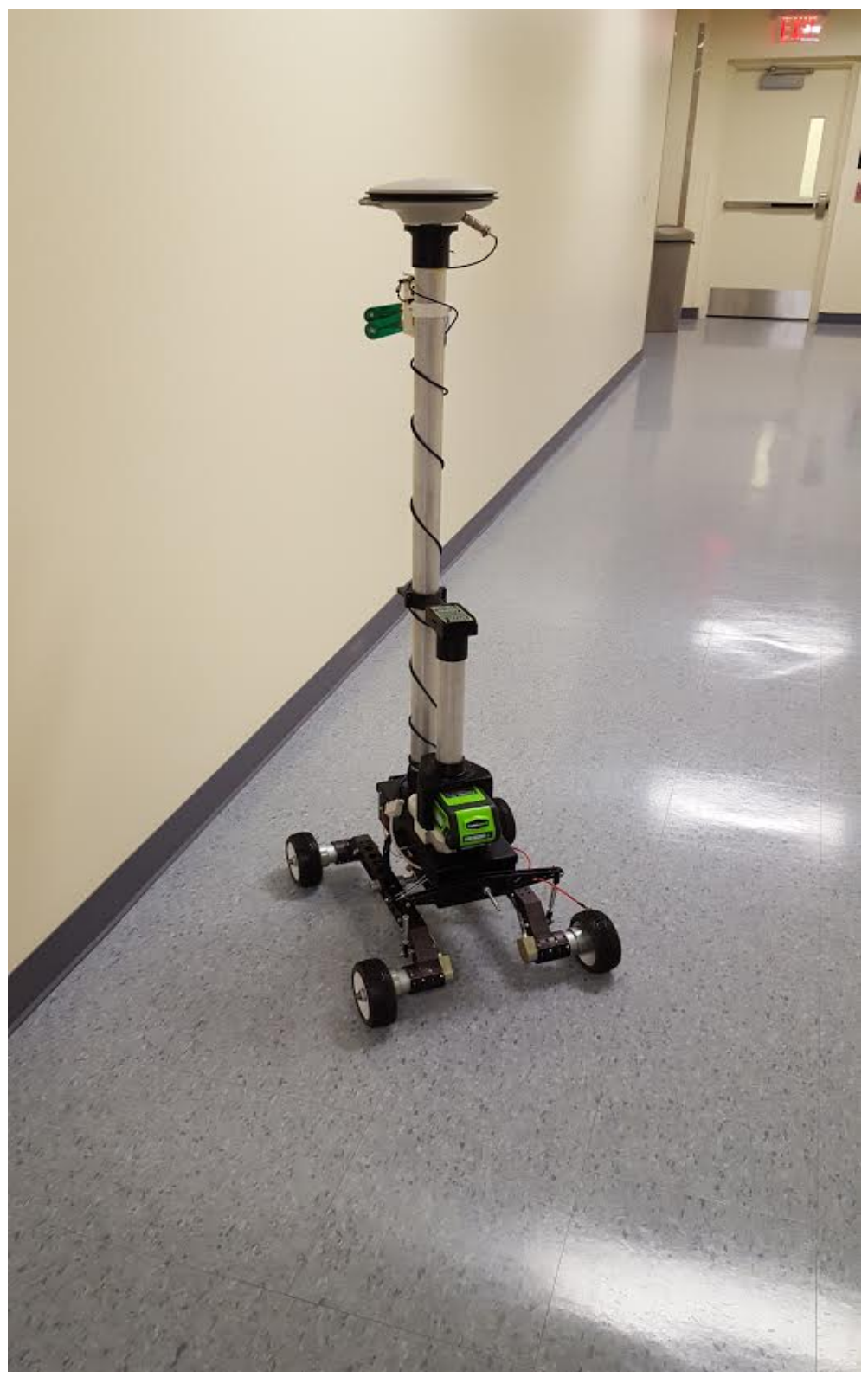

Figure 8.1: Rover with Visible UWB Radio and GPS Antennas at the Top of Mast

\subsection{Quadrotor}

The quadroter was designed to be the second moving platform required to properly test the UKF in an experimental environment and was equipped and tested by Victor Sivaneri. The quadrotor has a payload consisting of Analog Devices 16488AMLZ IMU with tri-axial magnetometer [48], a Time Domain PulsON P-410 UWB ranging radio [43], and a Ublox GPS reciever and Antenna [49]. In a similar fashion to the rover, a Netburner MOD54415 [46] and accompanying avionics board designed 
by Scott Harper is used to collect and store IMU and magnetomter data on an SD card, while the Ublox GPS data is stored on a seperate SD card. The quadrotor autopilot is latest generation WVU avionics with functionality comparable to systems described in [50].

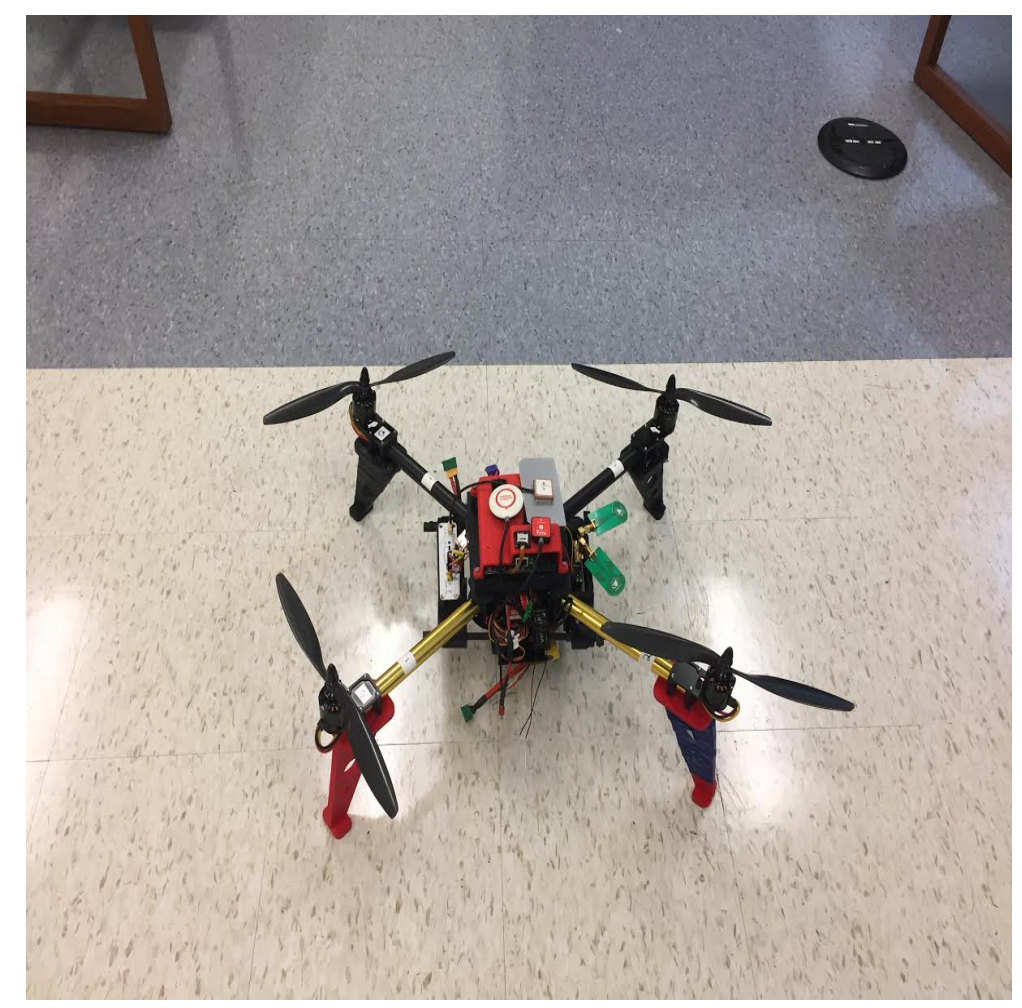

Figure 8.2: Quadrotor with Visible UWB Radio and GPS Antennas

\subsection{UWB Protocol}

The UWB ranging radio system uses a master slave protocol. The rover interfaces with its UWB to command the sensor to send a range request to the UWB on the quadrotor, in our setup this occurs at a frequency of $5 \mathrm{~Hz}$. The UWB on the quadrotor is passively waiting to receive this request and once it is received it sends the ranging data back to the master UWB on the rover. No data from the UWB on the quadrotor is collected, in fact the only connection from the quadrotor to its UWB is used to power the sensor. The only data recorded in this system is the range information received by the rover master UWB. Figure 8.3 shows a Time Domain P-410 with antennas attached and attached to rover mast. 


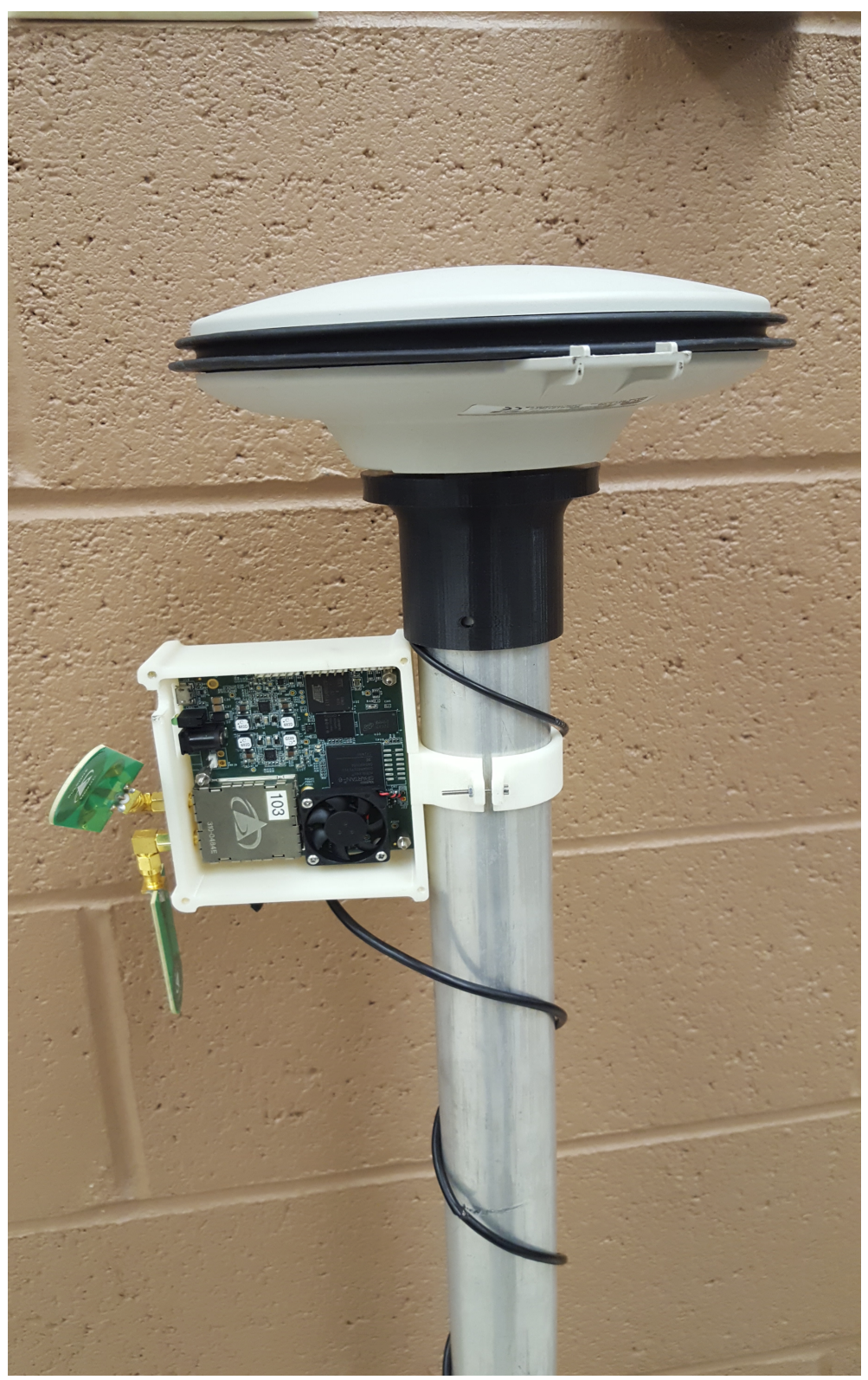

Figure 8.3: UWB Ranging Radio and NovAtel Pinwheel Antenna on Rover Mast

\subsection{System Overview}

These two vehicles equipped with the sensor payloads described provide a way to collect real IMU and ranging radio data to test the UKF. While magnetometer data on both platforms may be available in the future for now this measurement will be excluded.

In addition to the platforms described in the sections above a base station GPS will be setup nearby while the experiments are in session. The base station will allow a differential GPS solution 
for both platforms, which is preferable as differential GPS is known to eliminate satellite and atmospheric errors present in standard GPS solutions. These GPS measurements can be fused with the on-board IMUs in a Kalman filter to create a baseline truth for position and attitude for the experiment. Having a reliable truth for any experiment is important for analyzing results. In this case this truth solution will also be used to simulate the computer vision solution utilized in the $\mathrm{UKF}$ as the platforms do not have the equipment required to provide these measurements.

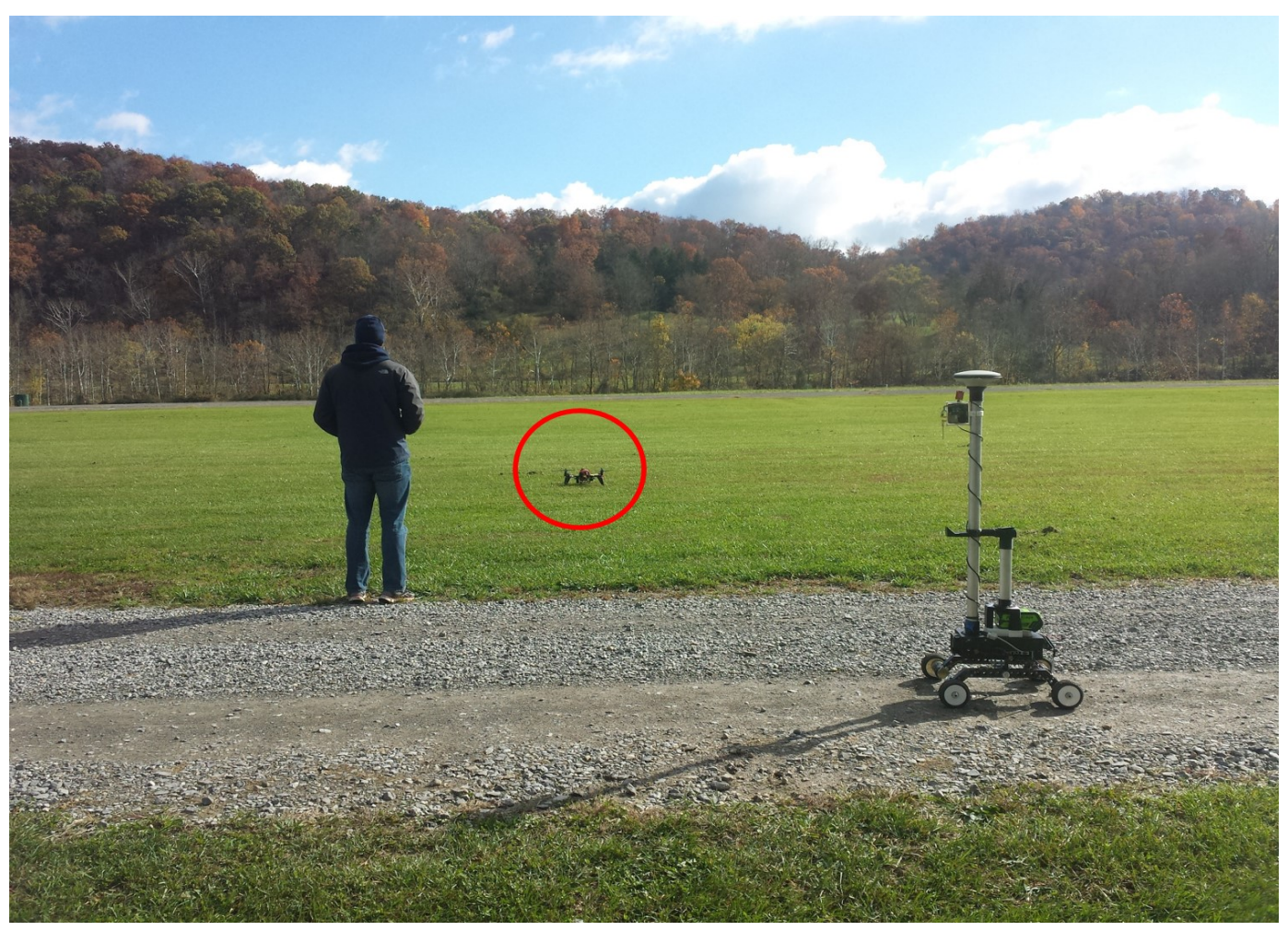

Figure 8.4: Flight Testing Photo before Quadrotor Takeoff 


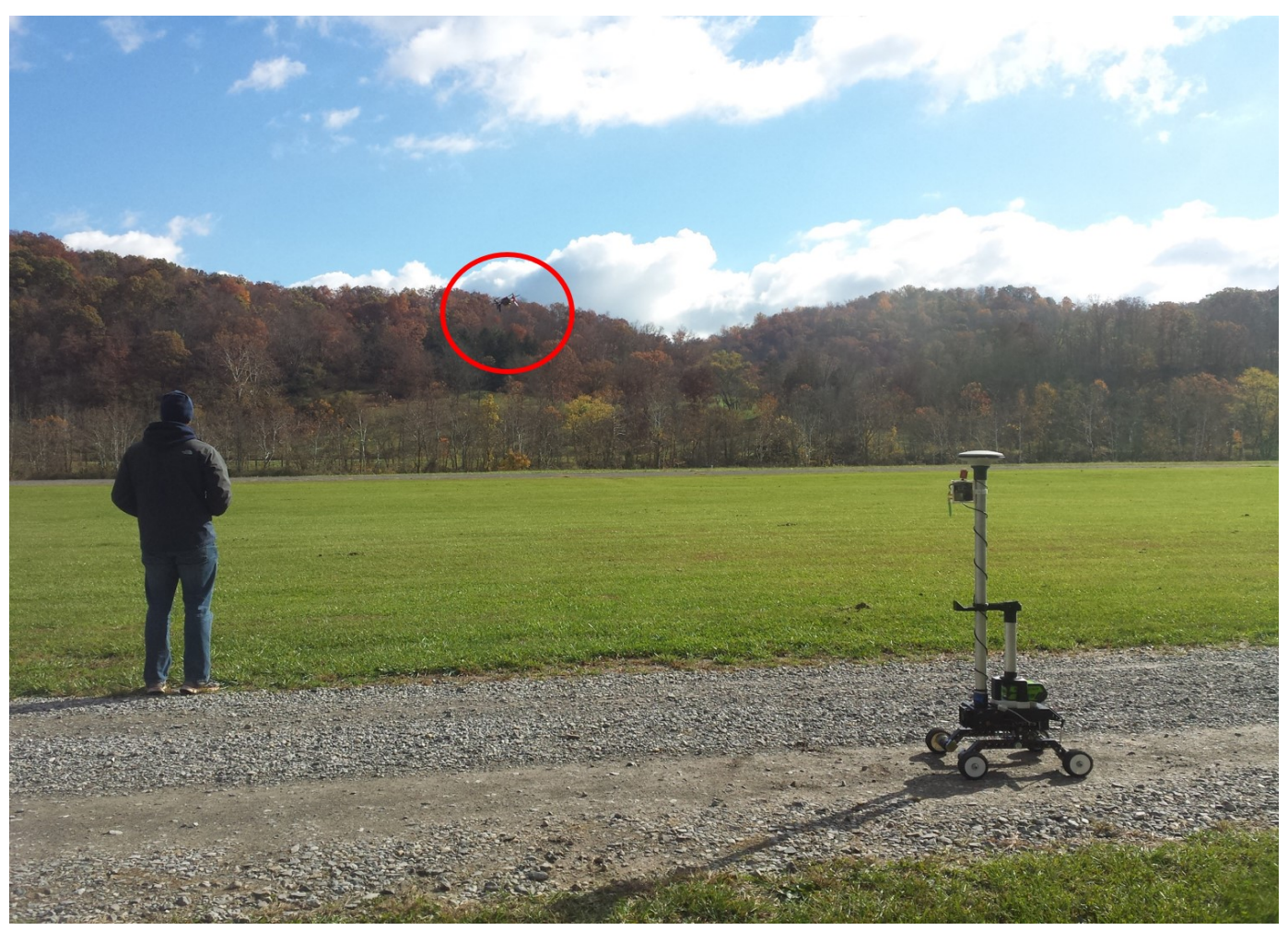

Figure 8.5: Flight Testing Photo with Quadrotor in Flight 


\section{Chapter 9}

\section{Conclusion}

This thesis presented an UKF formulation constructed for the estimation of relative pose between two UAVs operating in GNSS-denied environments with specific and identical sensor payloads. This formulation was then implemented in MATLAB to be tested for accuracy of the algorithm during a short simulated flight as well as test the formulations sensitivity to flight dynamics, initial error, and on-board sensor characteristics across multiple simulations. The algorithm is shown to greatly out perform an IMU only dead reckoning solution in a matter of seconds. The overall Monte Carlo results yielded a median RSS Position error of less than 9 meters and a median RSS attitude error of less than 0.5 degrees when every epoch of all 500 simulated 8 minute flights where considered. The most notable results of the sensitivity analysis shows the importance of using an IMU that is classified as Tactical Grade or higher particularly for attitude accuracy. The frequency UWB ranging radio and computer vision measurements had significant impact on positioning. While the frequency and quality of magnetometer measurements had significant impact on both position and attitude estimation. A more extensive analysis of an individual flight simulation revealed the significant impact that errors in computer vision unit vector measurements had on the smoothness of the positioning solution, especially when the total distance between the platforms was large. Finally, the design of experimental platforms for testing the algorithm outside of simulation was presented.

Future work on this project is mainly focused on the collection of data on the experimental platform described in Chapter 8 in order to analyze the accuracy of the UKF in a real environment. This process would include the testing and implementation of a more realistic UWB ranging radio error model and methods for properly aligning data from the two platforms with respect to time. Additionally, the presented simulation could explore the use of altimeters to determine the effect the 
sensor measurements would have on the position solution. Methods for modeling computer vision error and identifying outliers in the measurements could also be considered. Finally, the effect of augmenting the UKF could be tested particularly with regards to more effectively representing error in the computer vision rotation measurements. 


\section{Bibliography}

[1] Ryan, A., Zennaro, M., Howell, A., Sengupta, R., and Hedrick, J. K., "An overview of emerging results in cooperative UAV control," Decision and Control, 2004. CDC. 43rd IEEE Conference on, Vol. 1, IEEE, 2004, pp. 602-607.

[2] Fenwick, J. W., Newman, P. M., and Leonard, J. J., "Cooperative concurrent mapping and localization," Robotics and Automation, 2002. Proceedings. ICRA'02. IEEE International Conference on, Vol. 2, IEEE, 2002, pp. 1810-1817.

[3] Madhavan, R., Fregene, K., and Parker, L. E., "Distributed cooperative outdoor multirobot localization and mapping," Autonomous Robots, Vol. 17, No. 1, 2004, pp. 23-39.

[4] Beard, R. W., McLain, T. W., Nelson, D. B., Kingston, D., and Johanson, D., "Decentralized cooperative aerial surveillance using fixed-wing miniature UAVs," Proceedings of the IEEE, Vol. 94, No. 7, 2006, pp. 1306-1324.

[5] Richards, M. D., Whitley, D., Beveridge, J. R., Mytkowicz, T., Nguyen, D., and Rome, D., "Evolving cooperative strategies for UAV teams," Proceedings of the 7th annual conference on Genetic and evolutionary computation, ACM, 2005, pp. 1721-1728.

[6] Groves, P. D., Principles of GNSS, inertial, and multisensor integrated navigation systems, Artech House, 2013.

[7] Conte, G. and Doherty, P., "Vision-based unmanned aerial vehicle navigation using georeferenced information," EURASIP Journal on Advances in Signal Processing, Vol. 2009, 2009, pp. 10.

[8] Blösch, M., Weiss, S., Scaramuzza, D., and Siegwart, R., "Vision based MAV navigation in unknown and unstructured environments," Robotics and automation (ICRA), 2010 IEEE international conference on, IEEE, 2010, pp. 21-28. 
[9] Weiss, S., Scaramuzza, D., and Siegwart, R., "Monocular-SLAM-based navigation for autonomous micro helicopters in GPS-denied environments," Journal of Field Robotics, Vol. 28, No. 6, 2011, pp. 854-874.

[10] Artieda, J., Sebastian, J. M., Campoy, P., Correa, J. F., Mondragón, I. F., Martínez, C., and Olivares, M., "Visual 3-d slam from uavs," Journal of Intelligent and Robotic Systems, Vol. 55, No. 4-5, 2009, pp. 299-321.

[11] Caballero, F., Merino, L., Ferruz, J., and Ollero, A., "Vision-based odometry and SLAM for medium and high altitude flying UAVs," Unmanned Aircraft Systems, Springer, 2008, pp. 137161.

[12] Leishman, R. C., McLain, T. W., and Beard, R. W., "Relative navigation approach for visionbased aerial GPS-denied navigation," Journal of Intelligent \& Robotic Systems, Vol. 74, No. 1-2, 2014, pp. 97-111.

[13] Johnson, E. N., Calise, A. J., Watanabe, Y., Ha, J., and Neidhoefer, J. C., "Real-time visionbased relative aircraft navigation," Journal of Aerospace Computing, Information, and Communication, Vol. 4, No. 4, 2007, pp. 707-738.

[14] Valasek, J., Gunnam, K., Kimmett, J., Junkins, J. L., Hughes, D., and Tandale, M. D., "Visionbased sensor and navigation system for autonomous air refueling," Journal of Guidance, Control, and Dynamics, Vol. 28, No. 5, 2005, pp. 979-989.

[15] Sharma, R. and Taylor, C., "Cooperative Navigation of MAVs In GPS Denied Areas," Multisensor Fusion and Integration for Intelligent Systems, IEEE, 2008.

[16] Fosbury, A. M. and Crassidis, J. L., "Relative Navigation of Air Vehicles," Journal of Guidance, Control, and Dynamics, Vol. 31, No. 4, 2008, pp. 284-834.

[17] Gross, J. N., Gu, Y., and Rhudy, M. B., "Robust UAV Relative Navigation With DGPS, INS, and Peer-to-Peer Radio Ranging," Automation Science and Engineering, IEEE Transactions, Vol. 12, No. 3, 2015, pp. 935-944.

[18] Strader, J., Gu, Y., Gross, J., DePetrillo, M., and Hardy, J., "Cooperative Relative Localization for UAVs with Single Link Range Measurements," Proceedings of IEEE/ION Position Location and Navigation Symposium, IEEE/ION, 2016. 
[19] Hardy, J., Strader, J., Gross, J. N., Gu, Y., Keck, M., Douglas, J., and Taylor, C. N., "Unmanned aerial vehicle relative navigation in GPS denied environments," 2016 IEEE/ION Position, Location and Navigation Symposium (PLANS), IEEE, 2016, pp. 344-352.

[20] NovAtel, "IMU Errors and Their Effects," http://www.novatel.com/assets/Documents/ Bulletins/APN064.pdf, February 2014.

[21] "HG1930 Datasheet," https://aerospace.honeywell.com/products/ communication-nav-and-surveillance/inertial-navigation/ inertial-measurement-units/hg1930, 2015.

[22] Mayo, R. A., "Relative Quaternion State Transition Relation," Journal of Guidance, Control, and Dynamics, Vol. 2, No. 1, 1979, pp. 44-48.

[23] Blei, D. M., Ng, A. Y., and Jordan, M. I., "Latent Dirichlet Allocation," Jornal of Machine Learning Research, Vol. 3, 2003, pp. 993-1022.

[24] Lowe, D., "Distinctive image features from scale-invariant keypoints," International Journal of Computer Vision, Vol. 60, No. 2, 2004, pp. 91-110.

[25] Stewenius, H., Engels, C., and Nister, D., "Recent Developments on Direct Relative Orientation," ISPRS Journal of Photogrammetry and Remote Sensing, Vol. 60, No. 4, 2006, pp. 284294.

[26] Fischler, M. and Bolles, R., "Random Sample Consensus: A Paradigm for Model Fitting with Application to Image Analysis and Automated Cartography," Communications of the ACM, Vol. 24, No. 6, 1981, pp. 381-395.

[27] Solutions, V. E. N., "Magnetometer," http://www.vectornav.com/support/library/ magnetometer, Accessed Novemeber, 2016.

[28] Kalman, R. E., "A New Approach to Linear Filtering and Prediction Problems," Transactions of the ASME-Journal of Basic Engineering, Vol. 82, No. Series D, 1960, pp. 35-45.

[29] Simon, D., Optimal state estimation: Kalman, H infinity, and nonlinear approaches, John Wiley \& Sons, 2006.

[30] Julier, S. J. and Uhlmann, J. K., "New extension of the Kalman filter to nonlinear systems," AeroSense'97, International Society for Optics and Photonics, 1997, pp. 182-193. 
[31] Rhudy, M., Gu, Y., and Napolitano, M. R., "An analytical approach for comparing linearization methods in EKF and UKF," International Journal of Advanced Robotic Systems, Vol. 10, 2013.

[32] Rhudy, M., Gu, Y., Gross, J., Gururajan, S., and Napolitano, M. R., "Sensitivity analysis of extended and unscented Kalman filters for attitude estimation," Journal of Aerospace Information Systems, Vol. 10, No. 3, 2013, pp. 131-143.

[33] Gross, J. N., Gu, Y., Rhudy, M. B., Gururajan, S., and Napolitano, M. R., "Flight-test evaluation of sensor fusion algorithms for attitude estimation," IEEE Transactions on Aerospace and Electronic Systems, Vol. 48, No. 3, 2012, pp. 2128-2139.

[34] Gross, J., Gu, Y., Gururajan, S., Seanor, B., and Napolitano, M. R., "A comparison of extended kalman filter, sigma-point kalman filter, and particle filter in GPS/INS sensor fusion," AIAA Guidance, Navigation, and Control Conference, 2010, pp. 1-19.

[35] Rhudy, M., Gu, Y., Gross, J., and Napolitano, M., "Sensitivity analysis of EKF and UKF in GPS/INS sensor fusion," AIAA guidance, navigation, and control conference, 2011, p. 6491.

[36] Wan, E. A. and Van Der Merwe, R., "The unscented Kalman filter for nonlinear estimation," Adaptive Systems for Signal Processing, Communications, and Control Symposium 2000. ASSPCC. The IEEE 2000, Ieee, 2000, pp. 153-158.

[37] Simon, D., Optimal State Estimation: Kalman, H Infinity, and Nonlinear Approaches, Wiley, 2006.

[38] Crassidis, J. L. and Junkins, J. L., Optimal estimation of dynamic systems, CRC press, 2011.

[39] Watson, R., Sivaneri, V., and Gross, J., "Performance Characterization of Tightly-Coupled GNSS Precise Point Positioning Inertial Navigation within a Simulation Environment," Submitted to the 2016 AIAA Guidance Navigation and Control Conference, AIAA, 2016.

[40] GPSoft, "Satellite navigation TOOLBOX 3.0 User Guide," 2003.

[41] Gu, Y., Seanor, B., Campa, G., Napolitano, M. R., and Gururajan, S., Autonomous formation flight: design and experiments, INTECH Open Access Publisher, 2009.

[42] "ADIS16488AMLZ Datasheet," http://www.analog.com/media/en/ technical-documentation/data-sheets/ADIS16488.pdf, 2014.

[43] "P-410 Ultra Wideband Ranging and Communications Module," http://www.timedomain. com/datasheets/TD_DS_P410_RCM_FA.pdf, 2014. 
[44] "NovAtel OEM615 Datasheet," http://www.novatel.com/assets/Documents/Papers/ OEM615.pdf, 2016.

[45] "NovAtel GPS-702-GG Pinwheel Antenna Product Sheet," http://www.novatel.com/ assets/Documents/Papers/GPS701_702GG.pdf, 2016.

[46] "Netburner MOD5441X Data Sheet," http://www.netburner.com/products/core-modules/ mod5441x, February 2016.

[47] "Xbee RF Modem Product Sheet," https://www.sparkfun.com/datasheets/Wireless/ Zigbee/XBee-Datasheet.pdf, 2016.

[48] "ADIS16405AMLZ Datasheet," http://www.analog.com/media/en/ technical-documentation/data-sheets/ADIS16405.pdf, 2015.

[49] "u-blox GNSS EVK-M8 Product Sheet," https://www.u-blox.com/sites/default/files/ products/documents/EVK-7-8-M8_ProductSummary_\%28UBX-13005308\%29.pdf, 2016.

[50] Gu, Y., Barchesky, F., Chao, H., Gross, J., and Napolitano, M., Avionics design for a sub-scale fault-tolerant flight control test-bed, INTECH Open Access Publisher, 2012. 